\title{
Medium chain triglyceride diet reduces anxiety-like behaviors and enhances social competitiveness in rats
}

Fiona Hollis ${ }^{1}$, Ellen Siobhan Mitchell ${ }^{2}$, Carles Canto ${ }^{2}$, Dongmei Wang ${ }^{2}$, Carmen Sandi*1

1. Brain Mind Institute, School of Life Sciences, Ecole Polytechnique Federale de Lausanne (EPFL), CH1015 Lausanne Switzerland.

2. Nestlé Institute of Health Sciences SA, EPFL Innovation Park, CH-1015 Lausanne Switzerland.

*Correspondence to: Carmen.sandi@epfl.ch

Key words: mitochondria, oxidative phosphorylation, glucose transporter, glycolysis, hexokinase, social dominance, anxiety 


\section{ABSTRACT}

Medium-chain triglycerides (MCT) are emerging as unique dietary supplements potentially relevant to improve brain dysfunctions. MCT are converted into ketones and free medium chain fatty acids that, in the brain, are highly effective energy sources to mitochondria and potentially less harmful than glucose metabolism to neurons. Given the recently established link between mitochondrial dysfunction and high anxiety and depression, we set this study to investigate the effectiveness of an MCT-enriched diet to ameliorate anxiety- and depression-related behaviors in rats. Male rats were distributed into two groups, matched for their anxiety-like behaviors in the elevated plus maze. Each group was given either MCT-supplemented diet or an isocaloric control diet for fifteen days. Starting from the eighth day of diet, rats were exposed to different behavioral tests. MCT-fed rats showed reduced anxiety-like behaviors and enhanced social competitiveness, while their coping responses in the forced swim test or sociability were not affected by the treatment. When evaluated at the end of the two-week MCT diet, mitochondrial respiration was reduced in the MPFC while unchanged in the nucleus accumbens. In the mPFC, enzymes related to glycolysis and oxidative phosphorylation were also decreased by MCT diet, while proteins controlling glucose and glutamate transport were increased. Altogether, our findings strongly suggest the effectiveness of MCT diet to exert anxiolytic effects. In the brain, our results point to the MPFC as a brain region in which MCT supplementation improves transport and control of energy substrates. 


\section{Introduction}

Medium-chain triglycerides (MCT) are saturated fats consisting of six to ten carbon atoms linked into chains predominantly found in limited foods as coconut oil, palm kernel oil, and dairy fats. MCT provide fewer calories and are more rapidly absorbed and metabolized compared to long-chain triglycerides (LCT), whose longer length cause them to be stored as fat in the body. Consequently, MCT are increasingly being used in ketogenic diets as an alternative to the most frequently ketogenic diets composed of LCT. MCT can specifically affect brain function and are progressively used to improve neurobehavioral symptoms and cognitive function in clinical and preclinical studies of epilepsy (Gama et al., 2015; Packer et al., 2016), neurodegeneration (Costantini et al., 2008; Sharma et al., 2014; Zhao et al., 2012) and ageing (Balietti et al., 2010; Wang and Mitchell, 2016).

While both LCT and MCT are converted into ketones, beta hydroxybutyrate, acetone and acetoacetate in the liver, MCT is also converted into free medium chain fatty acids (MCFA). Ketones and MCFA are brain bioavailable via MCT and fatty acid transporters. In the brain, ketones serve primarily as fuel for neurons and astrocytes (Ebert et al., 2003). In contrast to glucose metabolism, ketones cause less oxidative stress when used as a mitochondrial energy source, and can bypass energy-consuming steps of the glycolytic and TCA cycle pathway (Prins and Matsumoto, 2014) Unlike LCT, MCFA rapidly cross the double mitochondrial membrane in the absence of carnitine (Williamson et al., 1968). Moreover, MCFA have been shown to affect both mitochondrial function and metabolism in cellular models (Hughes et al., 2014; Schönfeld and Wojtczak, 2016; Thevenet et al., 2016), and improve systemic energy metabolism in vivo (Zhao et al., 2012). Therefore, the emerging view is that ketones and MCFA may offer a means to improve energy metabolism efficiency without causing deleterious effects on neuronal function.

A potential application in which an MCT diet could be of benefit is anxiety. Emerging evidence points to the existence of mitochondrial dysfunction in individuals with high anxiety levels (Hovatta et al., 2010a; Tyrka et al., 2016; Wang et al., 2017), which is in line with data that individuals with mitochondrial disorders frequently display anxiety symptoms (Anglin et al., 2012; Morava and Kozicz, 2013). Mouse models of anxiety-like behavior display mitochondrial dysfunction at different stages of mitochondrial activity (Einat et al., 2005; Filiou et al., 2011). Importantly, high trait anxiety is a strong risk factor for developing stress-induced depression and anxiety disorders (Castro et al., 2012; Sandi et al., 2008; Sandi and Richter-Levin, 2009), and brain mitochondrial dysfunction is also emerging as an important hallmark of depression (Filipović et al., 2017; Gardner and Boles, 2011; Hovatta et al., 2010b; Marazziti et al., 2011; Wang et al., 2017). Several studies have evaluated the ability of LCTbased ketogenic diets to ameliorate anxiety and depression (Bostock et al., 2017; Kashiwaya et al., 2013; Sussman et al., 2015). However, to our knowledge, no study to date has examined the effects of a MCT-supplemented diet in the context of anxiety- and depression-related behaviors.

In humans, anxious subjects exhibit reduced competitiveness under stress (Goette et al., 2015), which can be predictive of a subordinate social status (Gilbert et al., 2009). Likewise, we have recently shown that high anxious rats show a propensity to become subordinate when paired against a low-anxious counterpart in a social dominance test (Hollis et al., 2015; van der Kooij et al., 2017). In the brain, this competitive disadvantage linked to high anxiety is associated with impaired mitochondrial function (i.e., decreased respiration, reduced protein content for the electron transfer chains I and II, and reduced ATP) in the nucleus accumbens (NAc), a brain region critically implicated in reward, motivation 
and exertion of effort (Hollis et al., 2015). Boosting mitochondrial function with nicotinamide in the NAc was able to increase social dominance (Hollis et al., 2015). Additionally, the medial prefrontal cortex (mPFC) has been implicated in social status and may also be subject to diet modulation (Wang et al., 2011). Mitochondrial function critically depends on substrate uptake and metabolism (Brand and Nicholls, 2011). Thus, the type of energy supply may have direct consequences for mitochondrial function by affecting the availability of substrates for oxidative phosphorylation, membrane potential, and proton motive force.

Therefore, here, we aimed to investigate the effectiveness of an MCT diet to decrease anxiety- and depression-related behaviors in adult, male Wistar rats. In addition, we aimed to assess the potential of the MCT diet to ameliorate social competitiveness and associated mitochondrial function in specific brain regions associated with social competition and anxiety.

\section{Materials and Methods}

\subsection{Animals}

Adult male Wistar rats, aged 2-3 months, were used for all experiments. Animals were individually housed in polypropylene cages $(57 \times 35 \times 20 \mathrm{~cm})$ with abundant pine bedding in a temperature- $(23 \mathrm{C})$ and light- $(0700-1900 \mathrm{~h})$ controlled room. All animals had ad libitum access to standard food and water. Upon arrival to the facility, animals were allowed to habituate to the vivarium for one week and were then handled for $2 \mathrm{~min} / \mathrm{d}$ during 3 days prior to the start of all experiments. All experiments were performed with the approval of the Cantonal Veterinary Authorities (Vaud, Switzerland) and carried out in accordance with the European Communities Council Directive of 22 September 2010 (2010/63/EU).

\subsection{Behavioral Tests}

All behavioral manipulations were performed during the light phase by experimenters blind to treatment groups. All efforts were made to minimize the number of animals while maintaining statistical rigor.

\subsubsection{Elevated Plus Maze}

Prior to the dietary intervention, one hundred two animals were tested for anxiety-related behavior in the EPM as previously described (Hollis et al., 2015). Lighting was maintained at 15-16 lux in the open arms and 5-7 lux in the closed. Depending on the amount of time spent on the open arm animals were classified as high- (HA, $\leq 5 \%$ open arm duration) intermediate (IA, 6-20\% open arm duration) or lowanxious ( $L A, \geq 20 \%$ open arm duration). Before and in between testing the apparatus was cleaned with a $5 \%$ EtOH solution. Three animals that fell off the maze during the procedure were omitted from the study. After EPM testing, rats were further subdivided into control and MCT feeding groups with similar average anxiety levels. In total, the group sizes were as follows: $H A-C O N=14 ; I A-C O N=18 ; L A-C O N=17$; $H A-M C T=15 ;$ IA-CON=18; LA-CON=17.

\subsubsection{MCT dietary treatment and behavior testing schedule}

Following group separation according to the EPM scores, one group (MCT-treated) was given a diet of $5 \%$ medium chain triglycerides (40:60 of octanoic acid triglyceride and decanoic triglyceride) and while 
the second group was given a control diet ( $5 \%$ sunflower oil) via specially formulated animal chow with traditional fat, carbohydrate and protein ratios (5\% fat-sunflower oil, 35\% starch, $3.5 \%$ crude fiber, $18 \%$ protein; Kliba Diet AIN93-G) similar to standard chow diets for 15 days. The duration was chosen based on previous evidence of rapid behavioral responses to short-term MCT supplementation (de Almeida Rabello Oliveira et al., 2008). Food was weighed daily to allow the calculation of food intake. Animals were left undisturbed for the first week of dietary exposure. On the $8^{\text {th }}$ day, animals were evaluated for different behaviors using the following sequence of tests: light-dark box test, social dominance test, and social preference test. The experimental diet was continued throughout the behavioral testing, with two-day intervals of rest in between tests.

Finally, in order assess MCT effects on learned helplessness behavior, rats (10 from each treatment, matched for overall anxiety levels) were also assessed for forced swim test immobility for two consecutive days. After 15 days of dietary intervention, animals were euthanized by decapitation and brains collected for biochemical and metabolic analyses. See Figure $1 \mathrm{~A}$ for visual depiction of the schedule.

\subsubsection{Social preference test}

Animals were submitted to the social exploration test twice. The first test took place three days after phenotyping in the EPM to ensure that there were no baseline differences in social preference prior to the diet initiation (data not shown). Animals were tested again on the $12^{\text {th }}$ day of dietary treatment to examine the effects of the MCT diet on social preference behavior.

The social preference test was performed in a rectangular, three-chambered box that included a central compartment where the rat was initially placed. Thereafter, retractable doors were removed and the rat could explore the left and right-compartment for $10 \mathrm{~min}$. The left- and right-compartments were equipped with a floor-fixed transparent perforated Plexiglas cylinder that contained either an unfamiliar male juvenile rat or an unfamiliar object. The juveniles were between the ages of PND2532 to avoid aggressive behavior from the experimental animal. Juveniles were habituated to the apparatus and the chamber one day prior to testing to reduce their stress and anxiety levels. The time the rat spent sniffing either the juvenile (social target) or the novel object (inanimate target) was manually scored from videotapes by an experimenter who was blinded to the treatment groups.

\subsubsection{Light-dark box test}

On the $8^{\text {th }}$ day of exposure to the experimental diet, rats were tested in the light dark box. The apparatus and procedure have been previously described (Cordero and Sandi, 2007). One chamber was black (dark box) and kept at 20 lux while the other chamber was white (light box) with an illumination level of 160 lux. Before and in between testing, the apparatus was cleaned with a $5 \% \mathrm{EtOH}$ solution. Animals were introduced into the light compartment. Anxiety-like behavior was calculated from the percentage of time spent in a brightly lit area of an arena during the five-minute test.

\subsubsection{Social Dominance Test}

Rats were separated into pairs of rats where they were matched for weight and anxiety according to experiment. One group of animals were matched such that high anxious (HA) rats treated with control or MCT diet were paired against low anxious (LA) control-fed rats. Another group of rats were matched for weight and anxiety, such that diet was the only differing variable (IA CON vs IA MCT-treated. The 
experiments were designed to address the impact of diet on social dominance, while also investigating the MCT diet would improve social competitive behavior in HA rats.

As in our previous work (Cordero and Sandi, 2007; Hollis et al., 2015; van der Kooij et al., 2017), animals were marked on their body for identification and placed in pairs in a clean (neutral) cage without food or water for $20 \mathrm{~min}$. During the social dominance test, both rats displayed spontaneously offensive behavior, but this balance typically shifted in the favor of one animal towards the end of the test. Social dominance was estimated by summation of the total duration of offensive behaviors for each rat in the dyad (offensive upright, lateral threat and keeping-down behavior, as previously described (Koolhaus, 1980)). Auto-grooming and social investigation were also assessed to determine the specificity of the experiment diet effects on offensive behavior (Cordero and Sandi, 2007). Trials with infrequent offensive behavior (no rat displaying $>10 \mathrm{~s}$ of total offensive behavior) were excluded from analysis as the relative social dominance in these pairs cannot be reliably measured.

\subsubsection{Plasma ketones}

Blood was drawn from the tail vein on the $7^{\text {th }}$ day of diet and trunk blood was collected during euthanasia on the $15^{\text {th }}$ day. Blood was centrifuged, and plasma samples were analyzed for betahydroxybutyrate using a colorimetric kit (Cayman, US) according to the manufacturer's instructions.

\subsubsection{Western blots}

Tissues from medial prefrontal cortex and nucleus accumbens were homogenized in $200 \mathrm{~mL}$ Tris buffer (pH 7.4) containing $10 \%$ sucrose and protease inhibitors, sonicated, centrifuged (10000 g for $10 \mathrm{~min}$ at $4^{\circ} \mathrm{C}$ ) and kept at $-80^{\circ} \mathrm{C}$ until use. Protein concentration was evaluated for each sample by BCA assay

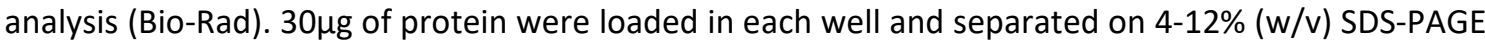
and transferred $(30 \mathrm{~V}, 12 \mathrm{~h}$ ) to nitrocellulose membranes (Whatman), which were then saturated (5\% BSA) and incubated with primary antibodies: alpha $2 \mathrm{Na} / \mathrm{K}$ ATPase subunit (1:1000; Abcam), complex I subunit 8 kDa [17C8E4E11] (1:1000; Abcam), Complex II 30 kDa subunit (1:2000; Invitrogen), excitatory amino acid transporter 2 (EAAT2, also call GLT-1) (1:1000; Abcam), GLUT-1 (1:500; Abcam), GSK3alpha/beta (1:1000; Life Technologies), GSK-3beta ser(9) (1:1000; Thermo Scientific), hexokinase-1 (1:2000; Abcam). Polyclonal antibodies against Actin (1:2000; Abcam) were incubated as loading controls. Secondary antibodies: Alexa 488 goat anti-rabbit (1:500; Life Technologies), Alexa 405 goat anti-mouse (1:500; Life Technologies) were incubated for $1 \mathrm{~h}$ prior to development using enhanced chemiluminescence (ECL system; Pierce). Bands were revealed and quantified using an Odyssey CLx imager (LI-COR). For measurement of cytoplasmic vs mitochondrial hexokinase, tissues were fractioned with a mitochondria isolation kit (Abcam), and then processed as described above.

\subsubsection{Mitochondria Respirometry}

On the $15^{\text {th }}$ day of experimental diet, animals were euthanized. The medial prefrontal cortex and nucleus accumbens were rapidly dissected out, weighed, and placed in a petri dish on ice with $2 \mathrm{~mL}$ of relaxing solution (2.8 mM Ca2K2EGTA, $7.2 \mathrm{mM} \mathrm{K2EGTA,} 5.8 \mathrm{mM}$ ATP, $6.6 \mathrm{mM} \mathrm{MgCl} 2,20 \mathrm{mM}$ taurine, $15 \mathrm{mM}$ sodium phosphocreatine, $20 \mathrm{mM}$ imidazole, $0.5 \mathrm{mM}$ dithiothreitol and $50 \mathrm{mM} \mathrm{MES}, \mathrm{pH}=7.1$ ) until further processing. Tissue samples were then gently homogenized in ice-cold respirometry medium (MiR05: $0.5 \mathrm{mM}$ EGTA, $3 \mathrm{mM} \mathrm{MgCl}$, $60 \mathrm{mM}$ potassium lactobionate, $20 \mathrm{mM}$ taurine, $10 \mathrm{mM}$ KH2PO4, $20 \mathrm{mM}$ HEPES, $110 \mathrm{mM}$ sucrose and 0.1\% (w/v) BSA, pH=7.1) with an Eppendorf pestle. 
Mitochondrial respiration rates were measured in $2 \mathrm{mg}$ samples of tissue at $37^{\circ} \mathrm{C}$ using high resolution respirometry (Oroboros Oxygraph 2K, Oroboros Instruments, Innsbruck, Austria), as previously described for other tissues (Hollis, 2015). A multi-substrate protocol was used to sequentially explore the various components of mitochondrial respiratory capacity. To measure the respiration due to oxidative phosphorylation, various substrates were used to activate specific complexes. Oxygen flux due to complex I activity $(\mathrm{Cl})$ was measured by the addition of ADP $(5 \mathrm{mM})$ to a solution containing malate $(2 \mathrm{mM})$, pyruvate $(10 \mathrm{mM})$ and glutamate $(20 \mathrm{mM})$, followed by the addition of succinate (10 $\mathrm{mM}$ ) to subsequently stimulate complex II $(\mathrm{Cl}+\mathrm{II})$. The maximal capacity of the electron transport system (ETS), was evaluated using the protonophore, carbonylcyanide 4 (trifluoromethoxy) phenylhydrazone (FCCP; successive titrations of $0.2 \mu \mathrm{M}$ until maximal respiration rates were reached). Oxygen consumption in the uncoupled state due to the activity of complex II was measured by inhibiting complex I with the addition of rotenone $(0.1 \mu \mathrm{M}$; ETS CII). Electron transport through complex III was inhibited by adding antimycin $(2 \mu \mathrm{M})$ to obtain the level of residual oxygen consumption (ROX) due to oxidating side reactions outside of mitochondrial respiration. The $\mathrm{O} 2$ flux obtained in each step of the protocol was normalized by the wet weight of the tissue sample used for the analysis and corrected for ROX.

\subsubsection{ATP Quantification}

ATP Determination Kit (A22066) from Molecular Probe was used to quantify ATP. Briefly, ATP-releasing buffer was freshly prepared (100mM Tris, 4mM EDTA, 1mM DTT and 1\% Triton 100). Cells from homogenized mPFC tissue were washed with ice-cold PBS and lysed in ATP-releasing buffer. Reaction Solution was prepared according to the manufacturer's instruction, avoiding light. 10 $\mathrm{ll}$ of samples were added into wells of a 96-well plate for luminescence measurement. The reaction was initiated by adding $100 \mu \mathrm{l}$ of Reaction Solution. Luminescence was measured after 10-20min incubation, and the concentrations were calculated based on a standard curve and normalized by the amount of protein of each sample.

\subsubsection{Mitochondrial Copy Number}

Mitochondrial copy number was performed on flash frozen tissue from CON- or MCT-treated rats euthanized under basal conditions. Tissue was placed in lysis buffer and DNA was extracted using DNeasy extraction kit (Qiagen). DNA concentrations were measured using the Nanodrop. Oligonucleotide primers for real-time quantitative PCR and tested to ensure all efficiencies were above 95\%. All primers were efficient between 97.8 and 99.7\%. Primers tested include: ND1, fwd TCCTCTTATCCGTCCTCCTAATAA; rev CAGGCGGGGATTAATAGTCA; ND4, fwd TTACACGATGAGGCAACCAA; rev GAGTGGGATGGAGCCAATTA; GAPDH, fwd AAACCCATCACCATCTTCCA; rev CCTCGAAGTACCCTGTGCAT. ND1 and ND4 are mitochondrial genes encoding complex I subunits, while GAPDH is a nuclear-encoded gene. Each reaction contained 200 $\mathrm{nM}$ each of the forward and reverse primers, SYBR Green PCR Master Mix (Applied Biosystems) and $10 \mathrm{ng}$ sample DNA in a $20 \mu \mathrm{l}$ reaction volume. The qPCR reactions were performed in triplicates in an ABI Prism 7900 Sequence Detection System (Applied Biosystems). The standard cycling conditions were $95^{\circ} \mathrm{C}$ for $5 \mathrm{~min}$, followed by 40 cycles of $95^{\circ} \mathrm{C}$ for $30 \mathrm{~s}$, and $60^{\circ} \mathrm{C}$ for $30 \mathrm{~s}$. Melt curves were generated at the end of the regular qPCR cycles. The comparative $\mathrm{Ct}$ method was used to determine raw copy numbers of ND1, ND4, and GAPDH. The copy numbers for ND1 and ND4 were then 
individually normalized to the nuclear-encoded gene, GAPDH. While neither subunit showed any significant difference, we present the data from ND1.

\subsubsection{Statistical Analyses}

All samples represent biological replicates. Data were analyzed using two-way ANOVAs where appropriate. Weight and plasma ketones were analyzed using a two-way repeated measures ANOVA, with time as the within-subjects factor and diet and trait anxiety as the between-subject factors. Within-pair amounts of behavior in the social dominance test were compared using paired two-tailed student's t-tests. In sets of data in which animals were matched for anxiety, relative social dominance scores were compared using one-sample t-tests against chance level (50\%). Mitochondrial respirometry experiments were performed in counterbalanced experimental blocks and analyzed using a generalized linear mixed model including a random effect of block. All data points are shown as group means with standard errors. Except for respirometry data, all data were analyzed using Prism version 5.01 and 7 (Graphpad software Inc, San Diego, CA). For respiration experiments, data were analyzed using SPSS statistical software version 21.0 (SPSS, Chicago, IL).

\section{RESULTS}

\subsection{Effect of MCT supplementation on weight, food intake, and plasma ketone levels}

We previously identified a link between trait anxiety and mitochondrial function, such that high anxious animals exhibited lower basal mitochondrial function that adversely affected their performance in a social competition (Hollis et al., 2015; van der Kooij et al., 2017). Thus, in order to account for any potential interactions with MCT, we classified animals according to their a priori basal anxiety levels on the Elevated Plus Maze prior to the start of the diet. The benefits of MCT diet supplementation have been attributed to the elevated ketone levels induced by medium chain fatty acid metabolism (Reger et al., 2004). As ketone levels are dependent on the amount of food intake, we first verified that there were no differences between groups in food consumption. Indeed, at the start of the diet, there were no significant differences between groups in weight (Figure 1B, $F(2,86)=$ $0.433 ; p=0.650$ ). Throughout the course of the diet, all groups exhibited significant weight gain (Figure $1 B, F(1,86)=248.9 ; p<0.0001)$, but there were no significant interactions with anxiety or treatment (Figure $1 \mathrm{~B}, \mathrm{~F}(2,86)=0.524 ; p=0.594)$. During the study, food consumption was also similar (Figure $1 \mathrm{C}$, treatment: $F(1,95)<0.0001 ; p=0.99$; interaction: $(F(2,95)=0.034 ; p=0.97)$.

To verify that the diet had successfully elevated ketones, plasma ketone body levels after 1 and 2 weeks of diet were evaluated. Ketone bodies are metabolized in the liver from fatty acid oxidation. Beta-hydroxybutyrate is formed upon fatty acid oxidation, a process typically upregulated when other energy sources such as glucose are low. Thus, beta-hydroxybutyrate is present in low levels in animals consuming typical chow diets, when most dietary fat is stored in fat cells. However, ketone production may be increased via high fat or ketogenic diets. Analysis of plasma ketone bodies, such as betahydroxybutarate (BHB), has been considered one of the more reliable measurements of a ketogenic diet (Carmant, 2008). Consumption of the MCT-supplemented diet led to a significant increase in the plasma ketone BHB compared to control diet rats (Figure 1D, Treatment effect $F(1,29)=8.836 ; p=$ 
$0.006)$, with no difference across time points $(F(1,29)=0.24 ; p=0.63)$, and no significant interaction between time and treatment $(F(1,29)=1.04 ; p=0.32)$. Moreover, there were no differences in plasma ketones according to trait anxiety, as there were no significant interactions between time and anxiety $(F(2,29)=0.57 ; p=0.57)$, or time, anxiety, and treatment $(F(2,29)=0.10 ; p=0.90)$. These results demonstrate that the MCT supplementation was capable of elevating plasma ketones in the absence of a ketogenic diet or caloric restriction.

\subsection{MCT supplementation decreases anxiety-like behavior}

During diet supplementation, we examined the effects of MCT on anxiety-like behaviors. To this end, animals were exposed to the light-dark box test after one week of MCT or CON diet. There was a significant effect of diet such that rats treated with MCT spent significantly more time in the lit area of the test compared to control-diet rats (Figure. $2 \mathrm{~A}, \mathrm{~F}(1,84)=4.83 ; p=0.03$ ), indicating that $\mathrm{MCT}$ decreased natural anxiety-like behavior.

We then investigated whether MCT treatment might decrease depressive-like behaviors. We exposed rats to the forced swim test and quantified the time spent immobile during the second day of the test. As trait anxiety extremes might have inherent differences in depressive-like behaviors, we only included a subset of rats who exhibited an intermediate anxious phenotype. Rats treated with MCT showed no differences in immobility compared to those receiving the control diet (Figure 2B, $t=1.07$; $\mathrm{df}=16 ; p=0.30)$.

\subsection{MCT supplementation enhances social dominance}

We recently identified a link between high anxiety and poor social competitive performance in rats (Hollis et al., 2015; van der Kooij et al., 2017). Given that MCT treatment reduced anxiety-like behavior, we hypothesized that MCT might act to boost competitive behavior. To test this hypothesis, we matched animals according to their a priori basal anxiety levels on the Elevated Plus Maze. Animals were weighed and carefully matched for body weight and condition, such that control- (CON) animals were matched against either CON or MCT-treated (MCT) animals and allowed to compete for twenty minutes. In animals matched for the same anxiety levels within the competing dyad, we found that $\mathrm{MCT}$ diet supplementation significantly enhanced social competitive behavior (Figure $2 \mathrm{Cleft}, \mathrm{t}=2.24$; $\mathrm{df}=12, p=0.04)$. We also examined the effect of MCT-diet on social competition in animals that differed in their a priori trait anxiety, as previously performed in (Hollis et al., 2015). As previously observed (Hollis et al., 2015; van der Kooij et al., 2017), we confirmed here that CON high anxious rats (HA) rats exhibited a significant disadvantage against CON low anxiety $(L A)$ rats (Figure $2 \mathrm{C}$ right, $\mathrm{t}=2.61$; $\mathrm{df}=6$; $p=0.04$ and Supplementary Figure 1A-D). Importantly, MCT diet supplementation significantly enhanced social competitive behavior in HA rats both, above chance level ( $t=4.2 ; d f=6, p=0.006)$ and above that of CON HA rats ( $t=4.55 ; \mathrm{df}=11, p=0.0007$ and Supplementary Figure $1 \mathrm{~A}-\mathrm{D})$, while having no effect on social investigation (HA MCT: $\mathrm{t}=0.056$; $\mathrm{df}=4 ; p=0.96$ ) or autogrooming (HA MCT: $\mathrm{t}=0.89$; $\mathrm{df}=$

$4 ; p=0.42$ ) behaviors (Supplementary Figure $1 \mathrm{E}-\mathrm{F}$ ). These data suggest that MCT can enhance social competitive behavior and reverse anxiety-related deficits on social dominance.

In a next experiment, we examined whether the effects of MCT diet on social competition could be related to nonspecific effects on animals' sociability by testing rats in the 3-chamber test for social preference. Overall, there were no significant effect of diet $(F(1,97)=1.61 ; p=0.20)$, and indeed, when taken together, both MCT- and control-diet rats exhibited a significant social preference for an 
unfamiliar juvenile rat over an inanimate object (CON: $t=10.94, d f=40, p<0.0001 ; \mathrm{MCT} ; \mathrm{t}=12.32, \mathrm{df}=$ $36, p<0.0001)$. However, there was a marginally significant effect of anxiety $(F(2,97)=2.62 ; p=0.076)$ and a significant interaction $(F(2,97)=3.22 ; p=0.044)$, with post hoc tests indicating that HA rats significantly increased their social preference following MCT diet supplementation (HA MCT vS HA CON, $p=0.028$ ), while IA and LA rats exhibited no change (Figure 2D; MCT vs CON, $p=0.98$ and $p=0.77$, respectively). These data suggest that the effects of diet on social competition may be related to increased social affiliation in HA rats.

\subsection{MCT supplementation affects mitochondrial function in a brain-specific manner}

Previous work has identified direct effects of medium chain fatty acid (MCFA) on mitochondrial biogenesis and activity (Hughes et al., 2014). Our previous work highlighted a functional link between brain mitochondrial function and social competitive performance (Hollis et al., 2015; van der Kooij et al., 2017), where mitochondrial function in the nucleus accumbens (NAc) was associated with trait anxiety and determined the outcome of a social competition. Furthermore, additional work directly implicates the synaptic strength in the medial prefrontal cortex (mPFC) in social dominance rank (Wang et al., 2011). As mitochondria have been linked with the regulation of synaptic strength (reviewed in (Vos et al., 2010)), mitochondrial function in the MPFC may be important for social competition. Thus, we hypothesized that MCT treatment would affect brain mitochondrial function and, therefore, at the end of the experiment we used high-resolution respirometry to assess mitochondrial respiration in the NAc and mPFC.

\subsubsection{MCT supplementation does not affect NAc mitochondrial function}

After 15 days of MCT treatment, when coupled respiration through complex I was stimulated in NAC homogenates by the addition of ADP, we found a significant effect of trait anxiety (Supplementary Figure 2; $\mathrm{Cl}: \mathrm{F}(1,12)=5.69 ; p=0.034)$. Stimulation of complex II with the addition of succinate similarly revealed a significant effect of trait anxiety $(\mathrm{Cl}+\mathrm{CII}: \mathrm{F}(1,12)=7.19 ; p=0.02)$, replicating our previous finding of inherent differences in NAc mitochondrial respiration according to trait anxiety (Hollis et al., 2015; van der Kooij et al., 2017). However, there was no effect of treatment $(\mathrm{Cl}: \mathrm{F}(1,12)=0.088 ; p=$ $0.77 ; \mathrm{Cl}+\mathrm{Cll}: \mathrm{F}(1,12)=0.024 ; p=0.88)$ or treatment $\mathrm{x}$ anxiety interaction $(\mathrm{Cl}: \mathrm{F}(1,12)=0.003 ; p=0.95$; $\mathrm{Cl}+\mathrm{ClI}: \mathrm{F}(1,12)=0.017 ; p=0.90)$. When respiration through the electron transport system was maximally induced (ETS), there was a significant effect of trait anxiety (ETS: $F(1,12)=8.53 ; p=0.013$ ), but no effect of treatment (ETS: $F(1,12)=0.39 ; p=0.55)$ or treatment $x$ anxiety interaction $(E T S: F(1,12)=$ $0.060 ; p=0.81$ ). When complex I was inhibited by rotenone for maximal electron transport system capacity (ETSCII), there was still a significant effect of anxiety (ETSCII: $F(1,12)=8.83 ; p=0.012$ ), but no effect of treatment (ETSCII: $\mathrm{F}(1,12)=0.34 ; p=0.57)$ or treatment by anxiety interaction (ETSCII: $F(1,12)=$ $0.38 ; p=0.55)$.

\subsubsection{MCT supplementation decreases MPFC mitochondrial function in high anxious animals}

In the $\mathrm{MPFC}$, for coupled mitochondrial respiration, there was a significant effect of both treatment (Figure 3A; $\mathrm{Cl}: \mathrm{F}(1,24)=7.75 ; p=0.010 ; \mathrm{Cl}+\mathrm{Cll}: \mathrm{F}(1,24)=11.67 ; p=.002)$ and anxiety $(\mathrm{Cl}: \mathrm{F}(1,24)=4.86$; $p=0.037 ; \mathrm{Cl}+\mathrm{Cll}: \mathrm{F}(1,24)=5.89 ; p=0.023)$, but no interaction $(\mathrm{Cl}: \mathrm{F}(1,24)=2.345 ; p=0.14 ; \mathrm{ClCll}: \mathrm{F}(1,24)=$ 2.86; $p=0.10)$. Post hoc analyses indicated that MCT treatment reduced respiration in high anxious animals significantly below that of high anxious CON animals (CON HA vs $\mathrm{MCT} \mathrm{HA:} \mathrm{Cl}: p=0.047 ; \mathrm{Cl}+\mathrm{Cll}$ : $p=0.03$ ) and to similar levels of low anxious animals (MCT HA vs MCT LA: Cl: 0.95; Cl+CII: $p=0.94 ;$ MCT 
HA vs CON LA: $\mathrm{Cl}: p=0.96 ; \mathrm{Cl}+\mathrm{Cll}: p=0.87)$. Treatment did not affect mitochondrial respiration in the mPFC of low anxious animals (CON LA vs MCT LA: $\mathrm{Cl}: p=0.75 ; \mathrm{Cl}+\mathrm{Cll}: p=0.54$ ). When the electron transport system was maximally induced (ETS), there was a significant effect of treatment (ETS: $F(1,25)=21.96 ; p<0.0001)$, no significant effect of trait anxiety (ETS: $F(1,25)=1.52 ; p=0.23$ ) and a trend for a treatment $x$ anxiety interaction (ETS: $F(1,25)=3.12 ; p=0.089$ ). The effects of MCT on respiration differences appear to be linked to complex I activity, as there were no significant effects of treatment or an interaction when complex I was inhibited with rotenone for maximal electron transport system capacity (Figure 3A; ETSCII: effect of treatment: $F(1,24)=2.18 ; p=0.15$; treatment $x$ anxiety: $F(1,25)=$ $0.078 ; p=0.78$ ). However, analysis of complex II-mediated maximal electron transport capacity revealed a significant effect of trait anxiety (ETSCII: $\mathrm{F}(1,25)=5.77 ; p=0.024)$.

Given the effects of MCT on mPFC mitochondrial function, we examined the protein expression of the mitochondrial complex subunits in this brain region (Supplementary Figure 3). Complex I protein levels were decreased in samples from MCT-treated animals as compared to CON, with a trend for a treatment effect (Figure 3B, $\mathrm{F}(1,14)=4.10 ; p=0.064)$ but no anxiety effect $(\mathrm{F}(1,14)=0.32 ; p=0.58)$. There were no significant effects of treatment for complex II protein levels (Figure 3C; Treatment: $F(1,19)=0.018 ; p=0.89 ;$ Anxiety: $F(1,19)=0.018 ; p=0.89)$, suggesting that the effects of MCT diet on mitochondrial function are related to complex I. Analysis of MPFC mitochondrial number by PCR of mitochondrial DNA revealed no significant effects of treatment on mitochondrial copy number (Figure 3D; Treatment: $F(1,20)=0.051 ; p=0.82$; Anxiety: $F(1,20)=0.77 ; p=0.39)$ levels, suggesting that the effects of MCT diet on respiration are not due to an increase in mitochondrial number. Lastly, we investigated the ATP levels in $\mathrm{MPFC}$ samples and found no significant effect of treatment between MCT and CON animals (Figure 3E; $F(1,18)=0.063 ; p=0.80$ ).

\section{MCT supplementation modulates the glutamate reuptake protein complex}

To better understand the changes in MPFC mitochondrial function induced by MCT diet, we examined metabolic signaling pathways in the MPFC at the protein level (Supplementary Figure 4A). Energy use in the brain is regulated via glucose and glutamate transport, as well as glycolytic enzymes and metabolites. Analysis of protein levels for GLUT1, a glucose transporter, identified a significant effect of MCT diet $t(F(1,16)=10.96 ; p=0.004)$ and a trend for an effect of anxiety $(F(1,16)=3.21 ; p=0.09)$ and interaction $(F(1,16)=3.21 ; p=0.09)$, where only MCT HA rats exhibited increased GLUT1 levels compared to CON HA (Figure $4 A ; p=0.006$ ) and LA rats were unaffected. Protein levels for GLT-1, a glutamate transporter located on astrocytes, were significantly increased, with a significant effect of treatment (Figure 4B; $F(1,15)=13.64 ; p=0.002)$ and a trend for an effect of anxiety $(F(1,15)=3.53 ; p=$ 0.08 ), with post-hoc tests indicating that the effect was mainly driven by HA rats (MCT HA vs CON HA: $p=0.012$; MCT LA vs CON LA: $p=0.12$ ). Glutamate uptake by GLT-1 occurs at the synapse and is dependent on sodium displacement through the NA/K-ATPase (Chatton et al., 2000; Pellerin and Magistretti, 1997; Rose et al., 2009), thus we also examined protein levels of NA/K-ATPase. Here we found that NA/K-ATPase protein levels mimicked those of GLT-1 - with a significant effect of treatment (Figure $4 C, F(1,15)=22.73 ; p=0.0002)$, no effect of anxiety, but a significant interaction $(F(1,15)=4.73$; $p=0.04$ ), mainly in HA animals (MCT HA vs CON HA: $p=0.0015$; MCT LA vs CON LA: $p=0.39$ ).

Hexokinase, which functions to sequester imported glucose and acts as the rate-limiting enzyme of glycolysis, couples cytosolic glycolysis to mitochondrial oxidative phosphorylation to produce ATP for glutamate reuptake by GLT-1/Na/K-ATPase complex (Genda et al., 2011). MCT diet induced a 
significant decrease in mPFC hexokinase levels, with a significant effect of treatment only-(Figure 4D; $F(1,16)=8.05 ; p=0.012)$, but no significant effects of anxiety or interaction. Hexokinase drives ETS oxidative phosphorylation via association to the outer mitochondrial membrane (Genda et al., 2011). This association is influenced by glycogen synthase kinase-3 phosphorylation (GSK-3), and aids in regulation of energy usage during times of metabolic stress (Pastorino and Hoek, 2008; Tanno et al., 2014). In our experiment, we found that MCT diet decreased GSK-3alpha/beta Ser9 phosphorylation in the mPFC with a treatment effect (Figure $4 \mathrm{E}, \mathrm{F}(1,15)=6.87, p=0.01$ ), though there were no significant effects of anxiety or interaction. Hexokinase typically localizes and binds to the outer mitochondrial membrane (Abu-Hamad et al., 2008; Wilson, 2003), but can also detach and remain unbound in the cytosol, shunting glucose to non-glycolytic pathways (Regenold et al., 2012; Rosa and de Cerqueira César, 2016). We therefore measured hexokinase protein levels in separated mitochondrial and cytosolic fractions. Here we found a significant effect of diet (Figure 4F and Supplementary Figure 4B; $F(1,8)=9.14 ; p=0.017)$, anxiety $(F(1,8)=5.62 ; p=0.045$, and an interaction $(F(1,8)=7.53 ; p=0.025)$ such that only MCT HA rats exhibited a significant increase in mitochondrial vs cytosolic hexokinase localization (MCT HA vs CON HA: $p=0.007$; MCT LA vs CON LA: $p=0.98$ ). The shift towards mitochondria-localized hexokinase may point to an increased regulation of energy levels. Taken together, our data point to a shift in glutamate reuptake in the MPFC that is related to anxiety and is specifically targeted by MCT.

\section{DISCUSSION}

Here, we present novel findings indicating the effectiveness of MCT diet to reduce anxiety-like behaviors and improve social competitiveness, a behavioral component that is deterred by high anxiety (Goette et al., 2015; Hollis et al., 2015; van der Kooij et al., 2017). However, our data does not reveal MCT diet-induced changes in two behaviors-coping responses in the forced swim test and social exploration-typically impaired in preclinical models of depression. We also show that MCT treatment leads to a reduction in mitochondrial respiration in the $\mathrm{MPFC}$, along with a reduction in complex I protein levels, and increased GLUT1, GLT1, Na/K ATPase and reduced hexokinase and reduced GSK $\alpha \beta$ phosphorylation.

We observed that 1 week of MCT treatment increased the time spent by rats in the anxiogenic, light compartment of the light-dark box test. This effect was observed in baseline anxiety, as our study did not target a model of genetic or stress-induced increased anxiety. Although high anxiety has been traditionally linked to dysregulation of neurotransmitters - such as GABA, serotonin and dopamineas well as neuropeptides, hormones and cytokines (Gold, 2015; Van Ameringen et al., 2000); altered mitochondrial function is currently emerging as a potential underlying mechanism of anxiety and considered as a target for novel treatments (Hovatta et al., 2010b; Tyrka et al., 2016). MitoQ, a compound that protects mitochondria against oxidative damage (Cochemé et al., 2007), was shown to exert anxiolytic effects in the dark-light test and altered glucose metabolism in animals that exhibited reduced anxiety (Nussbaumer et al., 2016).

In addition, our behavioral results show that MCT diet increases social dominance in a social competition test. Interestingly, this effect of diet is observed both in animals that show normal (i.e., animals that display intermediate anxiety levels when natural variation in a rat's percentage time in the open arm is considered) and in MCT-treated high anxious rats competing with control diet-treated 
low anxious rats. Our previous work has shown that high anxiety is associated to low social competitiveness and reduced mitochondrial respiration in the NAc. Recently, we reported that a low dose of the anxiolytic diazepam increases social competitiveness by boosting NAc mitochondrial function (van der Kooij et al., 2017). These findings align with observations that dominant mice display higher levels of energy-related metabolites in the NAc than subordinate mice (Larrieu et al., 2017). Although once again we confirm here that, under control conditions, high anxious rats display lower mitochondrial respiration in the NAc, our current data does not identify enhanced mitochondrial respiration in the NAc in MCT-treated animals. However, it is important to note that this aspect of mitochondrial function was examined following multiple testing of animals in tests involving different levels of stress, which might have affected different mitochondrial features (Hunter et al., 2016; Picard et al., 2015; Picard et al., 2016) masking a potential effect of MCT in NAc mitochondrial function.

Interestingly, we found decreased mPFC respiration and complex I protein content in MCT-treated animals. In addition, we report here for the first time greater mPFC respiration in high than in low anxious rats. The functional implications of these anxiety-related differences and MCT-treatment induced effects at the level of mitochondrial function in the MPFC are yet unknown. However, the convergent low mitochondrial respiration in the mPFC observed in both cases of low anxiety (i.e., animals low in this trait across a sample of outbred rats) and MCT-treated rats supports its potential relevance in this context and argues for the need of future mechanistic studies targeting this brain region. One possibility is that increased mitochondrial function in the MPFC might be indicative of hyperactivity in the region overall that exerts a negative influence on projections to other regions, such as the NAc. Thus, HA rats' lower NAc oxygen consumption may be due to prolonged PFC overactivity. Importantly, the mPFC-particularly, its glutamatergic transmission-has been implicated in the expression of social rank within a mouse social hierarchy (Wang et al., 2011; Zhou et al., 2017).

As to the potential mechanisms mediating the effects of MCT diet in brain and behavior, we confirmed increased plasma ketone levels without affecting total food consumption or body weight. However, MCFA derived from MCT have been proposed to alter mitochondria function by modulating citrate synthase and complex I activity (Hughes et al., 2014)). Thus, it is still unknown to what extent ketones are mediating observed behavioral and protein changes versus MCFA.

Remarkable differences were observed in terms of MCT modulation of energy substrate proteins in the mPFC. Interestingly, hyperactivity in this region has been associated with anxious mood (Arnold Anteraper et al., 2014; Bruhl et al., 2014). A possible anxiolytic mechanism of MCT is regulation of mPFC glutamate metabolism by ketones. Glutamate signaling is an important mechanism for mood disorders, as evidenced by the acute alleviation of depressive symptoms via administration of ketamine, a glutamate antagonist (Newport et al., 2015). Recently the anti-depressive effects of ketamine were shown to be partially mediated by GLT-1 (Liu et al., 2016). Moreover, blockade of GLT1 results in hyper-excitability and anxiety in animal models (John et al., 2015; Liu et al., 2016). Loss of Na-K ATPase is similarly linked to anxious behavior (Kirshenbaum et al., 2012; Riegel et al., 2009). The significant enhancement of GLT-1 and Na-K ATPase observed in our study in the MPFC of MCT-treated animals suggests increased capability to uptake excessive glutamate after the MCT diet in this brain region.

Importantly, both GLT-1 and Na-K ATPase are regulated by hexokinase 1 (Genda et al., 2011). In the mPFC, we found that hexokinase and complex I were decreased, while GLT-1 and Na/K ATPase 
increased by $\mathrm{MCT}$, indicating an uncoupling of glutamate activity to energy metabolism. Increased expression of cortical GLT-1 and $\mathrm{Na}^{+} \mathrm{K}^{-}$ATPase suggests greater astrocytic glutamate transport, but also increased coupling of glycolytic activity to mitochondrial function (Figure 5). Interestingly, the alterations in protein expression observed following MCT diet exposure did not impinge upon an a priori imbalance under basal conditions according to anxiety trait, as there were no significant differences in these proteins between high and low anxious rats on the CON diet. These findings suggest that the counteracting behavioral effects of the MCT diet might resulted from compensatory mechanisms helping to restore behavioral differences in the anxious rats, instead of reversing previously identified differences in NAc mitochondrial function. In addition, it should be noted that this study only focused on two brain regions implicated in social dominance and anxiety and certainly does not represent the state of the entire brain. Indeed, inputs from other regions that were not explored in this study may influence the activity in the MPFC, ultimately affecting behavior. One such potential region is the amygdala, which has reciprocal projections to the MPFC that can directly mediate anxiety and social behaviors (Felix-Ortiz et al., 2016). Although we previously showed that there are no differences between high and low anxious rats in amygdala mitochondrial respiration (Hollis et al., 2015), we cannot discard that exposure to the MCT diet may have exerted positive effects on mitochondrial function in the amygdala that may ultimately contribute to the anxiolytic and competitive behavioral responses.

Concurrent inhibition of mitochondrial respiration and glycolysis was reported to diminish extracellular glutamate clearance (Jackson et al., 2015). Prolonged exposure to MCT and ketones may be increasing glutamate transporter expression in a feedback response to decreased hexokinase and complex 1 activity. More efficient glutamate transport into astrocytes via GLT-1 may allow for enhanced control of glutamatergic activity, without the need for increased energy use. Indeed, the reduced mPFC respiration observed in MCT-treated animals was not reflected in the overall ATP levels, which were similar in all groups. Moreover, there were no group differences in mitochondrial copy number of mPFC tissues. Future experiments assessing the role of glutamate transporters in mediating mitochondrial efficacy may provide important insights to understand the link between high anxiety and MPFC hyperactivity.

In addition to enhancing PFC glutamate transport, MCT treatment may influence the coupling of glycolysis to oxidative phosphorylation. Increased mPFC hexokinase expression in mitochondrial vs cytoplasmic compartments in MCT-treated HA animals may be leading to more efficient energy use, as indicated by decreased oxidative respiration after MCT treatment. Hexokinase influences mitochondrial function via several pathways. When glycolysis rates go down in astrocytes, glutamate is increasingly shunted into the TCA cycle as an energy substrate (Melo et al., 2006)). Conversion of glutamate into alpha-ketoglutarate maintains the energy capacity of the astrocyte, but decreases TCA metabolites used by complex I. Additionally hexokinase association to the mitochondrial pore protein (VDAC) signals directly to the mitochondria to release metabolites that decrease complex 1 activity. Simultaneous decreases of mPFC glycolysis and complex 1 function lead to more efficient control of glutamate uptake and metabolism, and less cortical reactivity (Figure 5).

\section{Conclusions}

In conclusion, this study shows that MCT diet reduces anxiety-like behaviors and increases social competitiveness. Two weeks of MCT treatment modulated oxidative phosphorylation coupled with 
mitochondrial protein changes in the MPFC and in relation to individuals' basal anxiety. Furthermore, our date indicates the capacity of MCT to regulate glutamate signaling via altered transporters in the mPFC. Taken together, these data suggest improved transport and control of energy substrates in the mPFC by MCT treatment. Our work highlights the potential for MCT diets as a therapeutic tool for reducing anxiety, and related deficits in social competition in humans.

\section{Acknowledgments}

We would like to thank Ophalie Favrod for her assistance with the behavioral experiments. This project has been supported by grants from the Swiss National Science Foundation (31003A-152614; and NCCR Synapsy, grant no. 51NF40-158776), and intramural funding from the EPFL to CS. ESM, CC, and DW are employees of the Nestlé Institutes of Health Sciences SA. The funding sources had no additional role in study design, in the collection, analysis and interpretation of data, in the writing of the report or in the decision to submit the paper for publication.

\section{Conflict of Interest}

Ellen Siobhan Mitchell, Carles Canto, and Dongmei Wang are employees of Nestlé Institutes of Health Sciences SA. All other authors have no conflicts of interest to declare. 


\section{Figure legends}

Figure 1. MCT diet increased plasma ketone levels with no differences in food intake. A scheme depicts the timing of exposure to either MCT or CON diet and behavioral assay schedule; $\mathrm{X}$ denotes euthanasia (A). There were no differences in animal weight between control (CON) and medium chain triglyceride (MCT)-treated groups both at the start and finish of the diet intervention (B). Both CON and MCT groups consumed similar levels of chow (C). Plasma ketone levels were elevated in MCTtreated animals after one week of diet and remained increased until the end of the intervention, regardless of trait anxiety levels (D). All data are presented as means plus SEM. *, $p<0.05 ; n=14-$ 18/group.

Figure 2. MCT affects anxiety and social competitive behavior. Animals were tested for anxiety-like, social and depressive-like behaviors 1 and 2 weeks (respectively) after MCT diet exposure. After one week of diet exposure, MCT-treated animals spent significantly more time in the lit area of a light-dark box (A; $\mathbf{n = 1 4 - 1 8 / g r o u p ) . ~ M C T ~ d i e t ~ h a d ~ n o ~ e f f e c t ~ o n ~ i m m o b i l i t y ~ b e h a v i o r ~ i n ~ t h e ~ F o r c e d ~ S w i m ~ T e s t ~ i n ~}$ Intermediate anxious animals (B; $\mathbf{n = 9 / g r o u p ) . ~ М C T ~ d i e t ~ e n h a n c e d ~ s o c i a l ~ c o m p e t i t i v e ~ b e h a v i o r ~ i n ~}$ animals who were paired into dyads based on their a priori anxiety levels before diet intervention (C; $\mathbf{n = ~ 8 - 1 4}$ pairs), and increased social preference in high anxious (HA) animals only in the three-chamber social preference test ( $\mathbf{D} ; \mathbf{n = 1 4 - 1 8 / g r o u p ) . ~ A l l ~ d a t a ~ a r e ~ p r e s e n t e d ~ a s ~ m e a n s ~ p l u s ~ S E M . ~ * ~}, p<0.05$.

Figure 3. MCT diet reduces mitochondrial function in the medial prefrontal cortex. Mitochondrial respiration was assessed using an oxygraph on tissue homogenates from the medial prefrontal cortex (mPFC). In the mPFC, there was a significant effect of anxiety, such that control-fed high anxious rats exhibited higher levels of respiration compared to low anxious animals and only MCT-fed high anxious rats exhibited a reduction in respiration to that of low anxious levels ( $\mathbf{A} ; \mathbf{n}=\mathbf{8 / g r o u p}$ ). Western blots against mitochondrial complex proteins in the MPFC revealed alterations in protein levels. MCT diet marginally decreased complex I protein expression, with this effect targeting mainly the HA animals (B; $n=5$ /group). There were no differences in complex II protein expression when analyzed for treatment or anxiety (C; $\mathbf{n = 4 - 8 / g r o u p ) ~ A n a l y s i s ~ b y ~ q u a n t i t a t i v e ~ P C R ~ r e v e a l e d ~ n o ~ s i g n i f i c a n t ~ d i f f e r e n c e s ~}$ in mitochondrial DNA ratios ( $D ; n=5-6 / g r o u p)$. Similarly, quantification of ATP levels found no significant differences (E; $\mathbf{n = 5 - 6 / g r o u p ) . ~ A l l ~ d a t a , ~ e x c e p t ~ f o r ~ r e s p i r a t i o n ~ d a t a , ~ a r e ~ p r e s e n t e d ~ a s ~ m e a n s ~}$ plus SEM. Respiration data are presented as estimated marginal means \pm SEM of oxygen flux per $\mathrm{mg}$ tissue. ${ }^{\dagger}, \mathrm{p}<0.1 ; *, \mathrm{p}<0.05 ; * * *, \mathrm{p}<0.001$.

Figure 4. MCT diet alters protein levels to suggest a shift in glutamate reuptake in an anxietydependent manner. MCT diet significantly increased Glucose transporter 1 (GLUT1) protein levels, primarily in MCT-fed high anxious (HA) rats (A). The glutamate transporter 1 (GLT1) protein level was also increased in MCT-fed rats, primarily in MCT-fed HA rats (B). Levels of sodium-potassium ATPase (NA/K-ATPase) were also increased following MCT consumption, with HA rats exhibiting the increase (C). MCT diet significantly decreased hexokinase protein levels in low anxious (LA) rats (D). Phosphorylated glycogen synthase kinase-3 (pGSK-3) levels were also decreased following MCT diet in $\mathrm{HA}$ rats (E). The ratio of mitochondrial: cytosolic hexokinase was measured using antibodies against hexokinase in separated organelle fractions and significantly increased mitochondrial localization was observed in MCT-fed rats, primarily those who were HA (F). All data are presented as means plus SEM. $\dagger, \mathrm{p}<0.1 ;^{*}, \mathrm{p}<0.05 ;^{* *}, \mathrm{p}<0.01 ;{ }^{* * *}, \mathrm{p}<0.001 \mathrm{n}=5$ /group. 
Figure 5. Scheme depicting the interaction between the studied proteins. Glucose is taken up by astrocytes via the Glut1 transporter and is metabolized via glycolysis. When glucose is decreased, glutamate is shunted to the TCA cycle, generating intermediates for energy production via lactate and alpha-ketoglutarate that reduce metabolites for OXPHOS. With increased levels of hexokinase associated to the mitochondrial outer membrane, metabolites are released that decrease complex I activity. Additionally, medium chain fatty acids can enter and cross the mitochondrial outer membrane to additionally influence the TCA cycle. Meanwhile, increased levels of GLT-1 and NA-K ATPase lead to increased uptake of glutamate in astrocytes where it is converted to glutamine and transported to neurons. In neurons, glutamine is reversed to glutamate and packaged into synaptic vesicles, which are subsequently released into the synaptic cleft to bind to various synaptic and extrasynaptic receptors. [Glu=glutamate; Gln=Glutamine; HK1=hexokinase; TCA=The Citric acid cycle; Pyr=pyruvate; OXPHOS=oxidative phosphorylation] 


\section{Supplementary Figure legends}

Supplementary Figure 1. Individual behaviors for HA-CON and HA-MCT rats paired with LA-CON partners. HA-CON fed rats had significantly reduced offensive behavior when partnered to LA-CON rats that was reversed by MCT diet $(A)$ in: keeping down behavior (B) lateral Threat $(C)$ and offensive upright (D). Social investigation (E) and autogrooming (F) in both IA (left) and HA pairs were not different between MCT-fed and CON-fed animals.

Supplementary Figure 2. No effect of MCT diet on nucleus accumbens mitochondrial respiration. Mitochondrial respiration was assessed using an oxygraph on tissue homogenates from the nucleus accumbens (NAc). When mitochondrial respiration was analyzed according to the a priori (trait) anxiety levels of the animals, a significant effect of trait anxiety was observed in the NAc ( $\mathbf{n =}$ 4/group), such that control-fed high anxious rats had lower levels of respiration in comparison to their control-fed low anxious counterparts, though there was no effect of diet.

Supplementary Figure 3. Representative western blot images of medial prefrontal cortex complex I (A) and II (B) protein.

Supplementary Figure 4. Representative blots are included for each of the metabolic signaling pathway proteins investigated in the medial prefrontal cortex $(A)$ and for the mitochondrial vs cytosolic hexokinase protein levels (B). 


\section{References}

Abu-Hamad, S., Zaid, H., Israelson, A., Nahon, E., Shoshan-Barmatz, V., 2008. Hexokinase-I protection against apoptotic cell death is mediated via interaction with the voltage-dependent anion channel-1: mapping the site of binding. J Biol Chem 283, 13482-13490.

Anglin, R. E., Tarnopolsky, M. A., Mazurek, M. F., Rosebush, P. I., 2012. The psychiatric presentation of mitochondrial disorders in adults. J Neuropsychiatry Clin Neurosci 24, 394-409.

Arnold Anteraper, S., Triantafyllou, C., Sawyer, A. T., Hofmann, S. G., Gabrieli, J. D., Whitfield-Gabrieli, S., 2014. Hyper-connectivity of subcortical resting-state networks in social anxiety disorder. Brain Connect 4, 81-90.

Balietti, M., Casoli, T., Di Stefano, G., Giorgetti, B., Aicardi, G., Fattoretti, P., 2010. Ketogenic diets: an historical antiepileptic therapy with promising potentialities for the aging brain. Ageing research reviews 9, 273-279.

Bostock, E., Kirkby, K. C., Taylor, B. V., 2017. The current status of the ketogenic diet in psychiatry. Frontiers in psychiatry $8,43$.

Brand, M. D., Nicholls, D. G., 2011. Assessing mitochondrial dysfunction in cells. Biochem J 435, 297312.

Bruhl, A. B., Delsignore, A., Komossa, K., Weidt, S., 2014. Neuroimaging in social anxiety disorder-a meta-analytic review resulting in a new neurofunctional model. Neurosci Biobehav Rev 47, 260-280.

Carmant, L., 2008. Assessing ketosis: approaches and pitfalls. Epilepsia 49 Suppl 8, 20-22.

Castro, J. E., Diessler, S., Varea, E., Marquez, C., Larsen, M. H., Cordero, M. I., Sandi, C., 2012.

Personality traits in rats predict vulnerability and resilience to developing stress-induced depressionlike behaviors, HPA axis hyper-reactivity and brain changes in pERK1/2 activity.

Psychoneuroendocrinology 37, 1209-1223.

Chatton, J. Y., Marquet, P., Magistretti, P. J., 2000. A quantitative analysis of L-glutamate-regulated $\mathrm{Na}+$ dynamics in mouse cortical astrocytes: implications for cellular bioenergetics. Eur J Neurosci 12, 3843-3853.

Cochemé, H. M., Kelso, G. F., James, A. M., Ross, M. F., Trnka, J., Mahendiran, T., Asin-Cayuela, J., Blaikie, F. H., Manas, A.-R. B., Porteous, C. M., 2007. Mitochondrial targeting of quinones: therapeutic implications. Mitochondrion 7, S94-S102.

Cordero, M. I., Sandi, C., 2007. Stress amplifies memory for social hierarchy. Front Neurosci 1, 175184.

Costantini, L. C., Barr, L. J., Vogel, J. L., Henderson, S. T., 2008. Hypometabolism as a therapeutic target in Alzheimer's disease. BMC Neurosci 9 Suppl 2, S16.

de Almeida Rabello Oliveira, M., da Rocha Ataide, T., de Oliveira, S. L., de Melo Lucena, A. L., de Lira, C. E., Soares, A. A., de Almeida, C. B., Ximenes-da-Silva, A., 2008. Effects of short-term and long-term treatment with medium- and long-chain triglycerides ketogenic diet on cortical spreading depression in young rats. Neurosci Lett 434, 66-70. 
Ebert, D., Haller, R. G., Walton, M. E., 2003. Energy Contribution of Octanoate to Intact Rat Brain Metabolism Measured by 13C Nuclear Magnetic Resonance Spectroscopy. The Journal of Neuroscience 23, 5928-5935.

Einat, H., Yuan, P., Manji, H. K., 2005. Increased anxiety-like behaviors and mitochondrial dysfunction in mice with targeted mutation of the Bcl-2 gene: further support for the involvement of mitochondrial function in anxiety disorders. Behav Brain Res 165, 172-180.

Felix-Ortiz, A. C., Burgos-Robles, A., Bhagat, N. D., Leppla, C. A., Tye, K. M., 2016. Bidirectional modulation of anxiety-related and social behaviors by amygdala projections to the medial prefrontal cortex. Neuroscience 321, 197-209.

Filiou, M. D., Zhang, Y., Teplytska, L., Reckow, S., Gormanns, P., Maccarrone, G., Frank, E., Kessler, M. S., Hambsch, B., Nussbaumer, M., 2011. Proteomics and metabolomics analysis of a trait anxiety mouse model reveals divergent mitochondrial pathways. Biological psychiatry 70, 1074-1082.

Filipović, D., Todorović, N., Bernardi, R. E., Gass, P., 2017. Oxidative and nitrosative stress pathways in the brain of socially isolated adult male rats demonstrating depressive-and anxiety-like symptoms. Brain Structure and Function 222, 1-20.

Gama, I. R., Trindade-Filho, E. M., Oliveira, S. L., Bueno, N. B., Melo, I. T., Cabral-Junior, C. R., Barros, E. M., Galvão, J. A., Pereira, W. S., Ferreira, R. C., 2015. Effects of ketogenic diets on the occurrence of pilocarpine-induced status epilepticus of rats. Metabolic brain disease 30, 93-98.

Gardner, A., Boles, R. G., 2011. Beyond the serotonin hypothesis: mitochondria, inflammation and neurodegeneration in major depression and affective spectrum disorders. Prog Neuropsychopharmacol Biol Psychiatry 35, 730-743.

Genda, E. N., Jackson, J. G., Sheldon, A. L., Locke, S. F., Greco, T. M., O'Donnell, J. C., Spruce, L. A., Xiao, R., Guo, W., Putt, M., Seeholzer, S., Ischiropoulos, H., Robinson, M. B., 2011. Cocompartmentalization of the astroglial glutamate transporter, GLT-1, with glycolytic enzymes and mitochondria. J Neurosci 31, 18275-18288.

Gilbert, P., McEwan, K., Bellew, R., Mills, A., Gale, C., 2009. The dark side of competition: How competitive behaviour and striving to avoid inferiority are linked to depression, anxiety, stress and self-harm. Psychology and Psychotherapy: Theory, Research and Practice 82, 123-136.

Goette, L., Bendahan, S., Thoresen, J., Hollis, F., Sandi, C., 2015. Stress pulls us apart: Anxiety leads to differences in competitive confidence under stress. Psychoneuroendocrinology 54, 115-123.

Gold, P. W., 2015. The organization of the stress system and its dysregulation in depressive illness. Mol Psychiatry 20, 32-47.

Hollis, F., van der Kooij, M. A., Zanoletti, O., Lozano, L., Cantó, C., Sandi, C., 2015. Mitochondrial function in the brain links anxiety with social subordination. Proceedings of the National Academy of Sciences 112, 15486-15491.

Hovatta, I., Juhila, J., Donner, J., 2010a. Oxidative stress in anxiety and comorbid disorders. Neuroscience research 68, 261-275.

Hovatta, I., Juhila, J., Donner, J., 2010b. Oxidative stress in anxiety and comorbid disorders. Neurosci Res 68, 261-275. 
Hughes, S. D., Kanabus, M., Anderson, G., Hargreaves, I. P., Rutherford, T., O'Donnell, M., Cross, J. H., Rahman, S., Eaton, S., Heales, S. J., 2014. The ketogenic diet component decanoic acid increases mitochondrial citrate synthase and complex I activity in neuronal cells. J Neurochem 129, 426-433.

Hunter, R. G., Seligsohn, M., Rubin, T. G., Griffiths, B. B., Ozdemir, Y., Pfaff, D. W., Datson, N. A., McEwen, B. S., 2016. Stress and corticosteroids regulate rat hippocampal mitochondrial DNA gene expression via the glucocorticoid receptor. Proc Natl Acad Sci U S A 113, 9099-9104.

Jackson, J. G., O'Donnell, J. C., Krizman, E., Robinson, M. B., 2015. Displacing hexokinase from mitochondrial voltage-dependent anion channel impairs GLT-1-mediated glutamate uptake but does not disrupt interactions between GLT-1 and mitochondrial proteins. J Neurosci Res 93, 999-1008.

John, C. S., Sypek, E. I., Carlezon, W. A., Cohen, B. M., Ongur, D., Bechtholt, A. J., 2015. Blockade of the GLT-1 Transporter in the Central Nucleus of the Amygdala Induces both Anxiety and DepressiveLike Symptoms. Neuropsychopharmacology 40, 1700-1708.

Kashiwaya, Y., Bergman, C., Lee, J.-H., Wan, R., King, M. T., Mughal, M. R., Okun, E., Clarke, K., Mattson, M. P., Veech, R. L., 2013. A ketone ester diet exhibits anxiolytic and cognition-sparing properties, and lessens amyloid and tau pathologies in a mouse model of Alzheimer's disease. Neurobiology of aging 34, 1530-1539.

Kirshenbaum, G. S., Clapcote, S. J., Petersen, J., Vilsen, B., Ralph, M. R., Roder, J. C., 2012. Genetic suppression of agrin reduces mania-like behavior in $\mathrm{Na}+, \mathrm{K}+-$ ATPase alpha3 mutant mice. Genes Brain Behav 11, 436-443.

Larrieu, T., Cherix, A., Duque, A., Rodrigues, J., Lei, H., Gruetter, R., Sandi, C., 2017. Hierarchical Status Predicts Behavioral Vulnerability and Nucleus Accumbens Metabolic Profile Following Chronic Social Defeat Stress. Curr Biol 27, 2202-2210 e2204.

Liu, W. X., Wang, J., Xie, Z. M., Xu, N., Zhang, G. F., Jia, M., Zhou, Z. Q., Hashimoto, K., Yang, J. J., 2016. Regulation of glutamate transporter 1 via BDNF-TrkB signaling plays a role in the anti-apoptotic and antidepressant effects of ketamine in chronic unpredictable stress model of depression.

Psychopharmacology (Berl) 233, 405-415.

Marazziti, D., Baroni, S., Picchetti, M., Landi, P., Silvestri, S., Vatteroni, E., Catena Dell'Osso, M., 2011. Mitochondrial alterations and neuropsychiatric disorders. Curr Med Chem 18, 4715-4721.

Melo, T. M., Nehlig, A., Sonnewald, U., 2006. Neuronal-glial interactions in rats fed a ketogenic diet. Neurochem Int 48, 498-507.

Morava, E., Kozicz, T., 2013. Mitochondria and the economy of stress (mal)adaptation. Neurosci Biobehav Rev 37, 668-680.

Newport, D. J., Carpenter, L. L., McDonald, W. M., Potash, J. B., Tohen, M., Nemeroff, C. B., Biomarkers, A. P. A. C. o. R. T. F. o. N., Treatments, 2015. Ketamine and Other NMDA Antagonists: Early Clinical Trials and Possible Mechanisms in Depression. Am J Psychiatry 172, 950-966.

Nussbaumer, M., Asara, J. M., Teplytska, L., Murphy, M. P., Logan, A., Turck, C. W., Filiou, M. D., 2016. Selective mitochondrial targeting exerts anxiolytic effects in vivo. Neuropsychopharmacology $41,1751$. 
Packer, R. M., Law, T. H., Davies, E., Zanghi, B., Pan, Y., Volk, H. A., 2016. Effects of a ketogenic diet on ADHD-like behavior in dogs with idiopathic epilepsy. Epilepsy Behav 55, 62-68.

Pastorino, J. G., Hoek, J. B., 2008. Regulation of hexokinase binding to VDAC. J Bioenerg Biomembr 40, 171-182.

Pellerin, L., Magistretti, P. J., 1997. Glutamate uptake stimulates Na+,K+-ATPase activity in astrocytes via activation of a distinct subunit highly sensitive to ouabain. J Neurochem 69, 2132-2137.

Picard, M., McManus, M. J., Gray, J. D., Nasca, C., Moffat, C., Kopinski, P. K., Seifert, E. L., McEwen, B. S., Wallace, D. C., 2015. Mitochondrial functions modulate neuroendocrine, metabolic, inflammatory, and transcriptional responses to acute psychological stress. Proc Natl Acad Sci U S A 112, E6614-

6623.

Picard, M., Wallace, D. C., Burelle, Y., 2016. The rise of mitochondria in medicine. Mitochondrion 30, 105-116.

Prins, M. L., Matsumoto, J. H., 2014. The collective therapeutic potential of cerebral ketone metabolism in traumatic brain injury. Journal of Lipid Research 55, 2450-2457.

Regenold, W. T., Pratt, M., Nekkalapu, S., Shapiro, P. S., Kristian, T., Fiskum, G., 2012. Mitochondrial detachment of hexokinase 1 in mood and psychotic disorders: implications for brain energy metabolism and neurotrophic signaling. J Psychiatr Res 46, 95-104.

Reger, M. A., Henderson, S. T., Hale, C., Cholerton, B., Baker, L. D., Watson, G. S., Hyde, K., Chapman, D., Craft, S., 2004. Effects of beta-hydroxybutyrate on cognition in memory-impaired adults.

Neurobiol Aging 25, 311-314.

Riegel, R. E., Valvassori, S. S., Elias, G., Réus, G. Z., Steckert, A. V., de Souza, B., Petronilho, F., Gavioli, E. C., Dal-Pizzol, F., Quevedo, J., 2009. Animal model of mania induced by ouabain: evidence of oxidative stress in submitochondrial particles of the rat brain. Neurochemistry International 55, 491495.

Rosa, J. C., de Cerqueira César, M., 2016. Role of hexokinase and VDAC in neurological disorders. Current molecular pharmacology.

Rose, E. M., Koo, J. C., Antflick, J. E., Ahmed, S. M., Angers, S., Hampson, D. R., 2009. Glutamate transporter coupling to Na,K-ATPase. J Neurosci 29, 8143-8155.

Sandi, C., Cordero, M. I., Ugolini, A., Varea, E., Caberlotto, L., Large, C. H., 2008. Chronic stressinduced alterations in amygdala responsiveness and behavior--modulation by trait anxiety and corticotropin-releasing factor systems. Eur J Neurosci 28, 1836-1848.

Sandi, C., Richter-Levin, G., 2009. From high anxiety trait to depression: a neurocognitive hypothesis. Trends Neurosci 32, 312-320.

Schönfeld, P., Wojtczak, L., 2016. Short-and medium-chain fatty acids in energy metabolism: the cellular perspective. Journal of lipid research 57, 943-954.

Sharma, A., Bemis, M., Desilets, A. R., 2014. Role of medium chain triglycerides (Axona ${ }^{\circledR}$ ) in the treatment of mild to moderate Alzheimer's disease. American Journal of Alzheimer's Disease \& Other Dementias ${ }^{\circledR} 29,409-414$. 
Sussman, D., Germann, J., Henkelman, M., 2015. Gestational ketogenic diet programs brain structure and susceptibility to depression \& anxiety in the adult mouse offspring. Brain Behav 5, e00300.

Tanno, M., Kuno, A., Ishikawa, S., Miki, T., Kouzu, H., Yano, T., Murase, H., Tobisawa, T., Ogasawara, M., Horio, Y., Miura, T., 2014. Translocation of glycogen synthase kinase-3beta (GSK-3beta), a trigger of permeability transition, is kinase activity-dependent and mediated by interaction with voltagedependent anion channel 2 (VDAC2). J Biol Chem 289, 29285-29296.

Thevenet, J., De Marchi, U., Santo Domingo, J., Christinat, N., Bultot, L., Lefebvre, G., Sakamoto, K., Descombes, P., Masoodi, M., Wiederkehr, A., 2016. Medium-chain fatty acids inhibit mitochondrial metabolism in astrocytes promoting astrocyte-neuron lactate and ketone body shuttle systems. The FASEB Journal 30, 1913-1926.

Tyrka, A. R., Parade, S. H., Price, L. H., Kao, H. T., Porton, B., Philip, N. S., Welch, E. S., Carpenter, L. L., 2016. Alterations of Mitochondrial DNA Copy Number and Telomere Length With Early Adversity and Psychopathology. Biol Psychiatry 79, 78-86.

Van Ameringen, M., Mancini, C., Farvolden, P., Oakman, J., 2000. The neurobiology of social phobia: from pharmacotherapy to brain imaging. Curr Psychiatry Rep 2, 358-366.

van der Kooij, M. A., Hollis, F., Lozano, L., Zalachoras, I., Abad, S., Zanoletti, O., Grosse, J., Guillot de Suduiraut, I., Canto, C., Sandi, C., 2017. Diazepam actions in the VTA enhance social dominance and mitochondrial function in the nucleus accumbens by activation of dopamine D1 receptors. Molecular Psychiatry 23, 569.

Vos, M., Lauwers, E., Verstreken, P., 2010. Synaptic mitochondria in synaptic transmission and organization of vesicle pools in health and disease. Frontiers in synaptic neuroscience 2, 139.

Wang, D., Mitchell, E. S., 2016. Cognition and synaptic-plasticity related changes in aged rats supplemented with 8-and 10-carbon medium chain triglycerides. PLoS One 11, e0160159.

Wang, F., Zhu, J., Zhu, H., Zhang, Q., Lin, Z., Hu, H., 2011. Bidirectional control of social hierarchy by synaptic efficacy in medial prefrontal cortex. Science 334, 693-697.

Wang, X., Sundquist, K., Rastkhani, H., Palmer, K., Memon, A. A., Sundquist, J., 2017. Association of mitochondrial DNA in peripheral blood with depression, anxiety and stress- and adjustment disorders in primary health care patients. Eur Neuropsychopharmacol 27, 751-758.

Williamson, J. R., Browning, E. T., Scholz, R., Kreisberg, R. A., Fritz, I. B., 1968. Inhibition of fatty acid stimulation of gluconeogenesis by (+)-decanoylcarnitine in perfused rat liver. Diabetes 17, 194-208.

Wilson, J. E., 2003. Isozymes of mammalian hexokinase: structure, subcellular localization and metabolic function. J Exp Biol 206, 2049-2057.

Zhao, W., Varghese, M., Vempati, P., Dzhun, A., Cheng, A., Wang, J., Lange, D., Bilski, A., Faravelli, I., Pasinetti, G. M., 2012. Caprylic triglyceride as a novel therapeutic approach to effectively improve the performance and attenuate the symptoms due to the motor neuron loss in ALS disease. PLoS One 7, e49191. 
Zhou, T., Zhu, H., Fan, Z., Wang, F., Chen, Y., Liang, H., Yang, Z., Zhang, L., Lin, L., Zhan, Y., Wang, Z., $\mathrm{Hu}, \mathrm{H}$., 2017. History of winning remodels thalamo-PFC circuit to reinforce social dominance. Science $357,162-168$. 
A

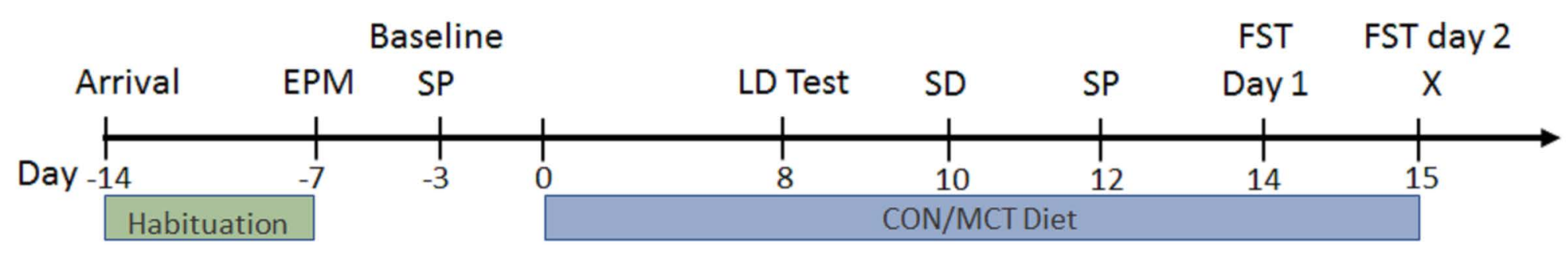

B

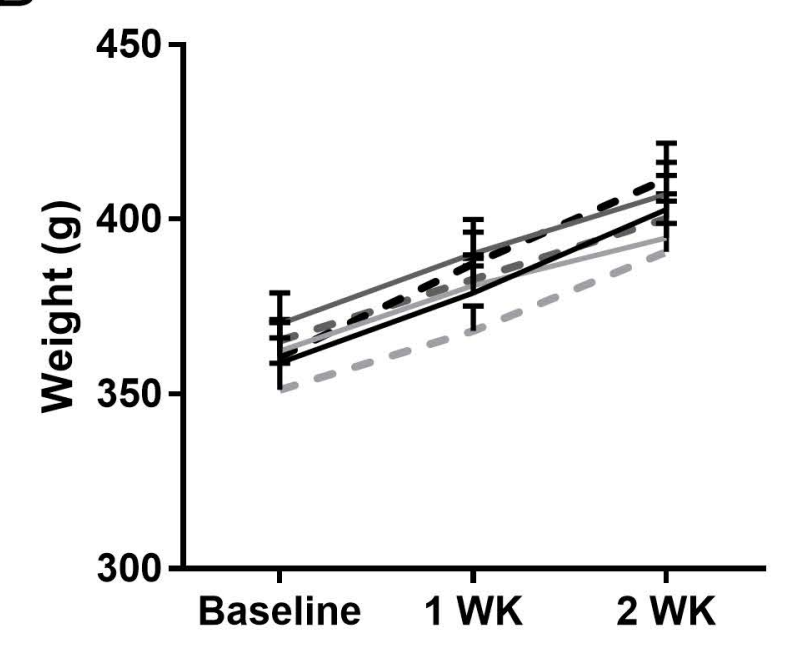

D

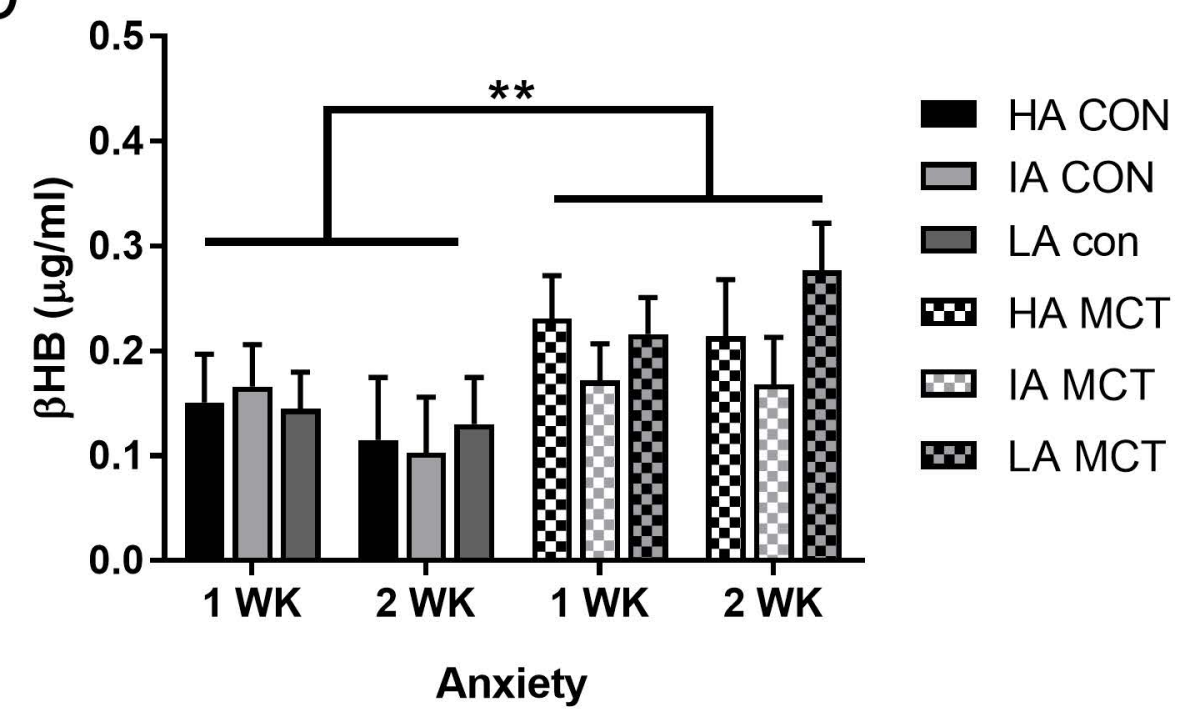

C

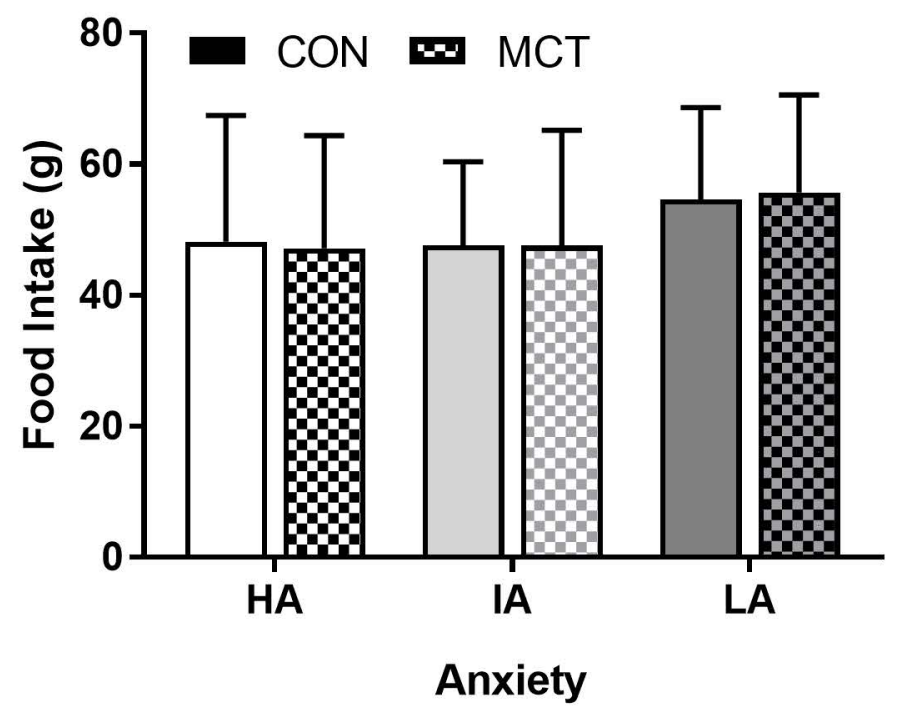


A
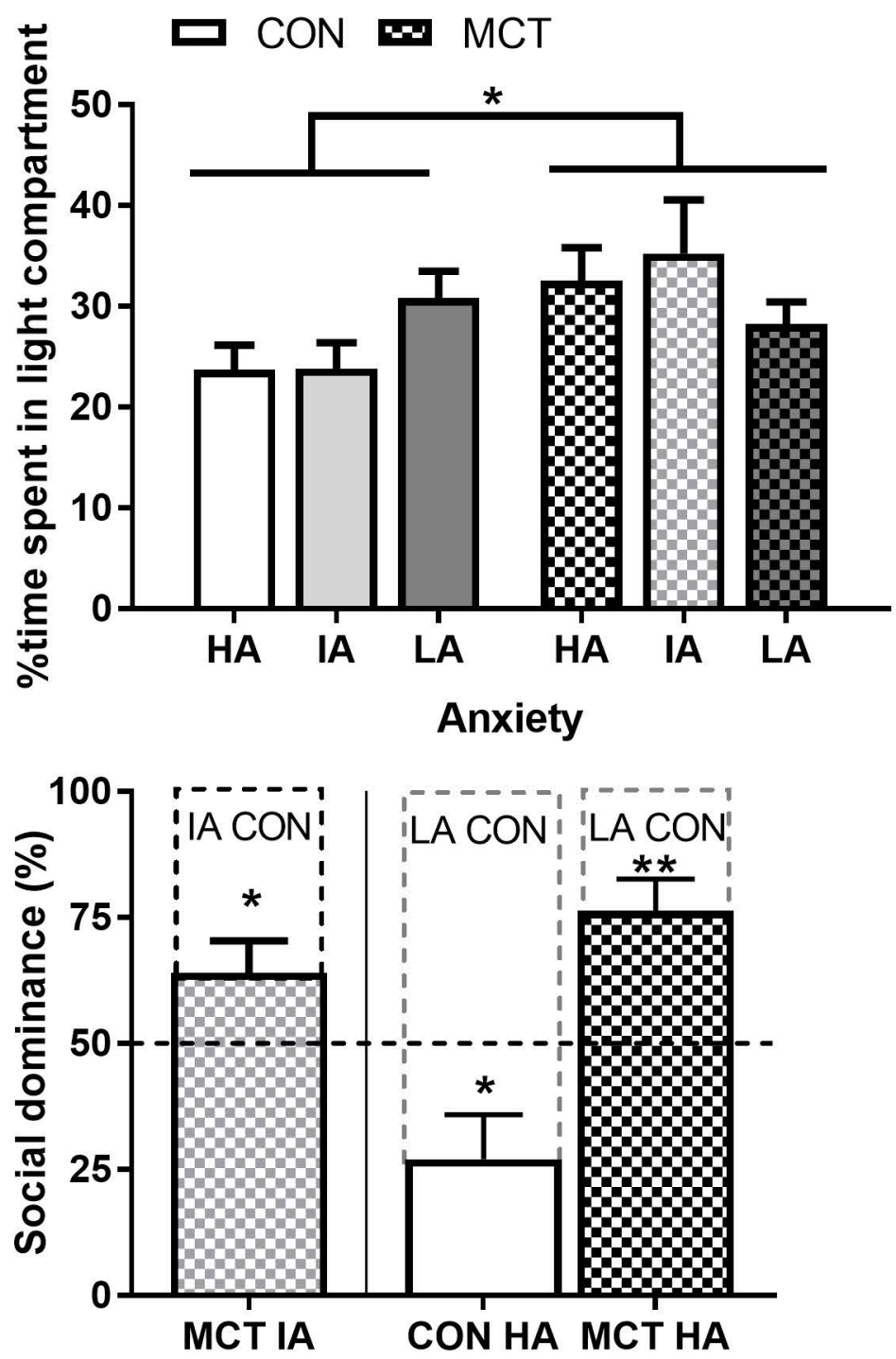

B

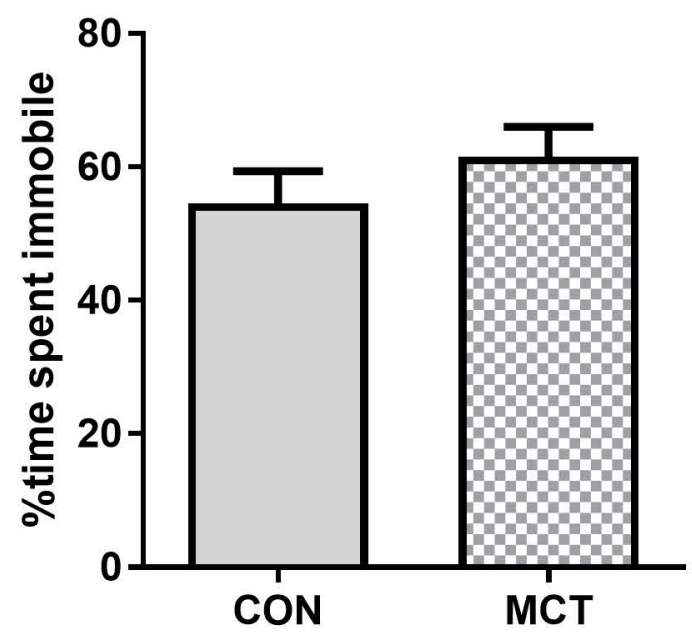

D

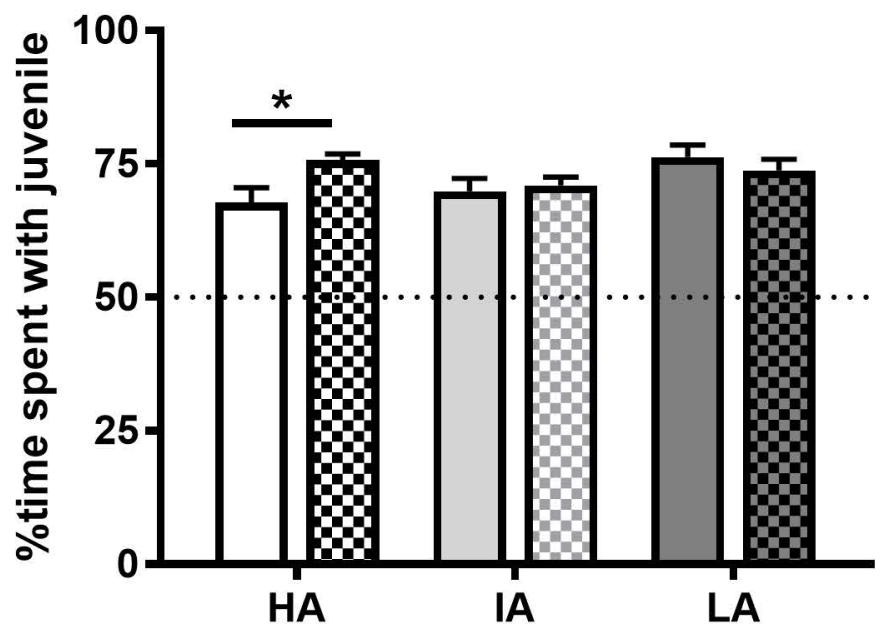


A

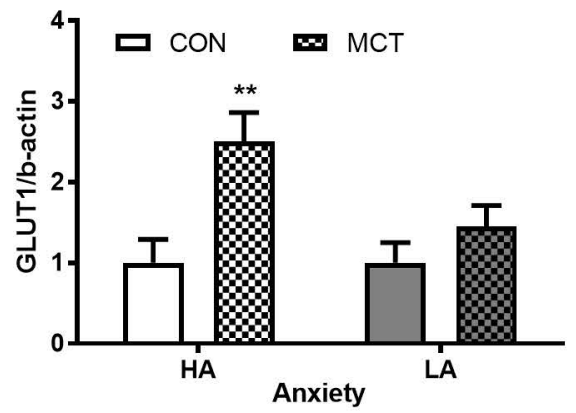

D

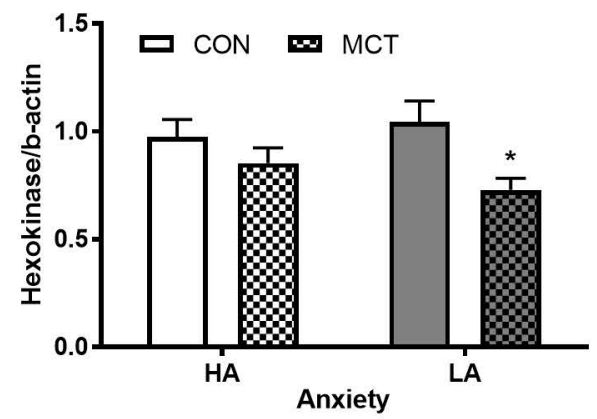

B

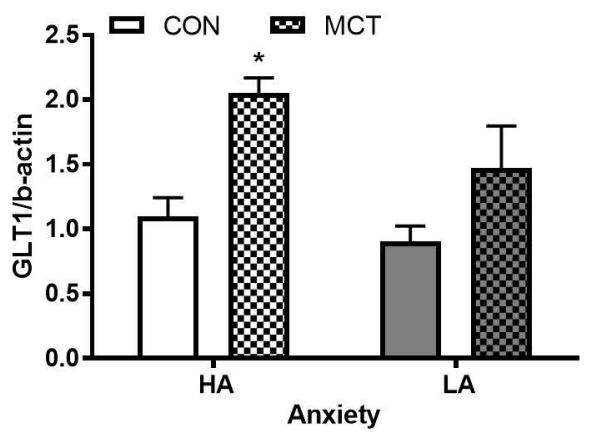

E

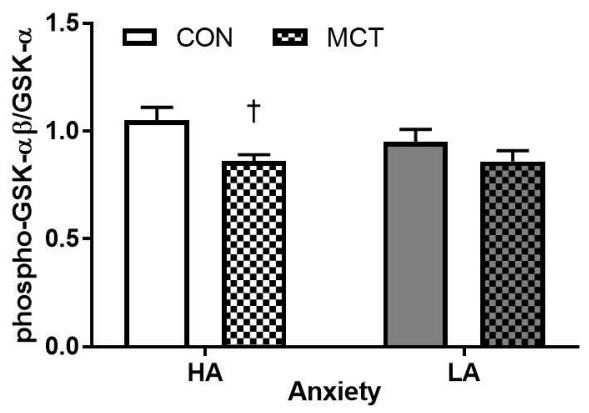

C

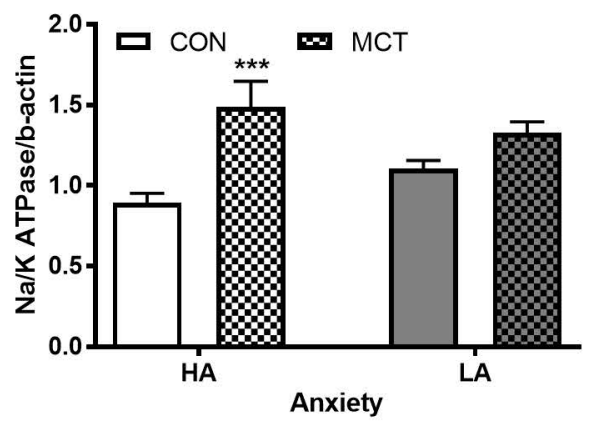

F

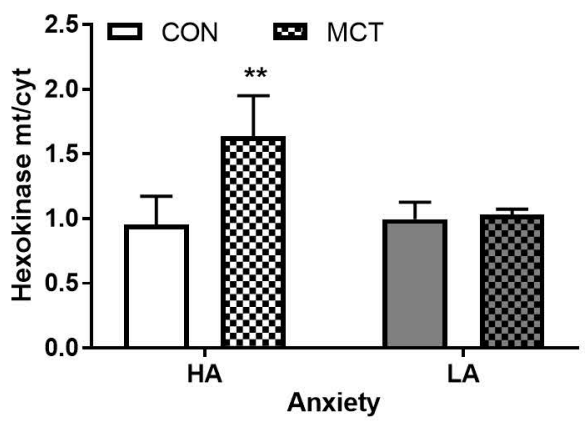




\section{Supplementary Information}

\section{Medium chain triglyceride treatment reduces anxiety-like behaviors and enhances social competitiveness in rats}

Fiona Hollis, Ellen Siobhan Mitchell, Carles Canto, Dongmei Wang, Carmen Sandi

\section{Supplementary Figures}
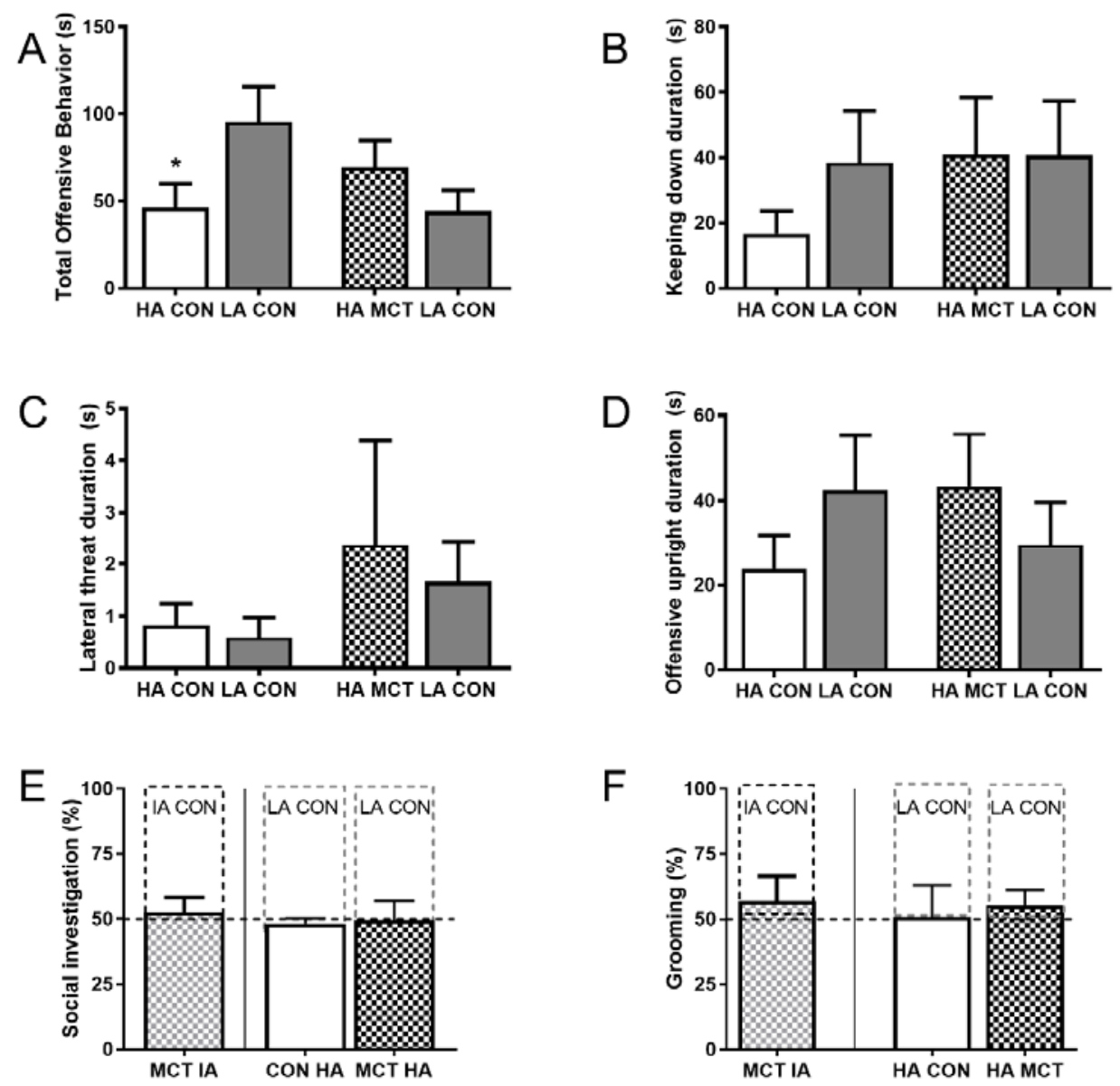

Supplementary Figure 1. Offensive behavior for HA-CON and HA-MCT rats paired with LA-CON partners. HA-CON fed rats had significantly reduced offensive behavior when partnered to LA-CON rats that was reversed by MCT diet (A) in: keeping down behavior (B) lateral Threat (C) and offensive upright (D). Social investigation (E) and autogrooming (F) in both IA (left) and HA pairs were not different between MCT-fed and CON-fed animals. 


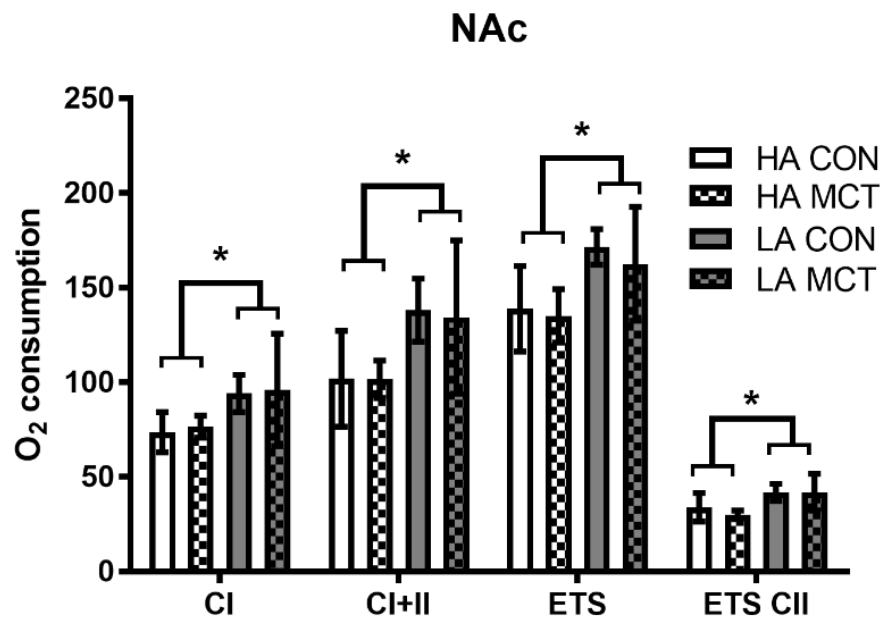

Supplementary Figure 2. No effect of MCT diet on nucleus accumbens mitochondrial respiration. Mitochondrial respiration was assessed using an oxygraph on tissue homogenates from the nucleus accumbens (NAc). When mitochondrial respiration was analyzed according to the a priori (trait) anxiety levels of the animals, a significant effect of trait anxiety was observed in the NAc ( $n=4 /$ group), such that control-fed high anxious rats had lower levels of respiration in comparison to their control-fed low anxious counterparts, though there was no effect of diet.

A

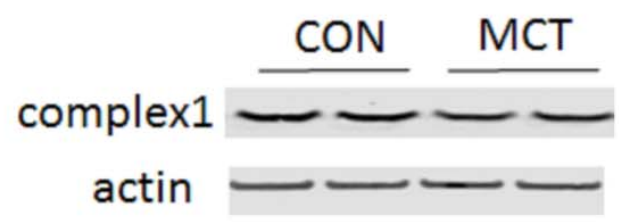

$\mathrm{B}$

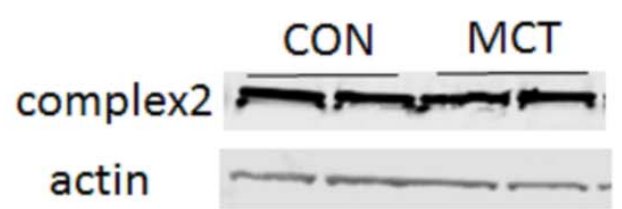

Supplementary Figure 3. Representative western blot images of medial prefrontal cortex complex I (A) and II (B) protein. 
A

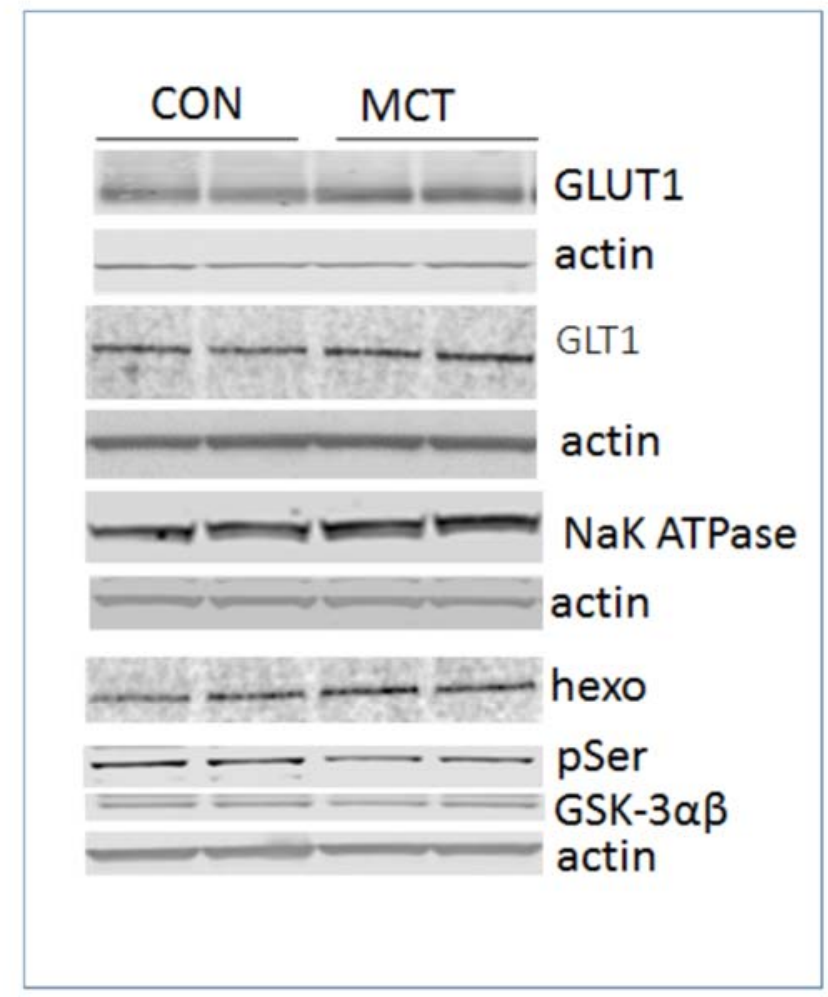

B

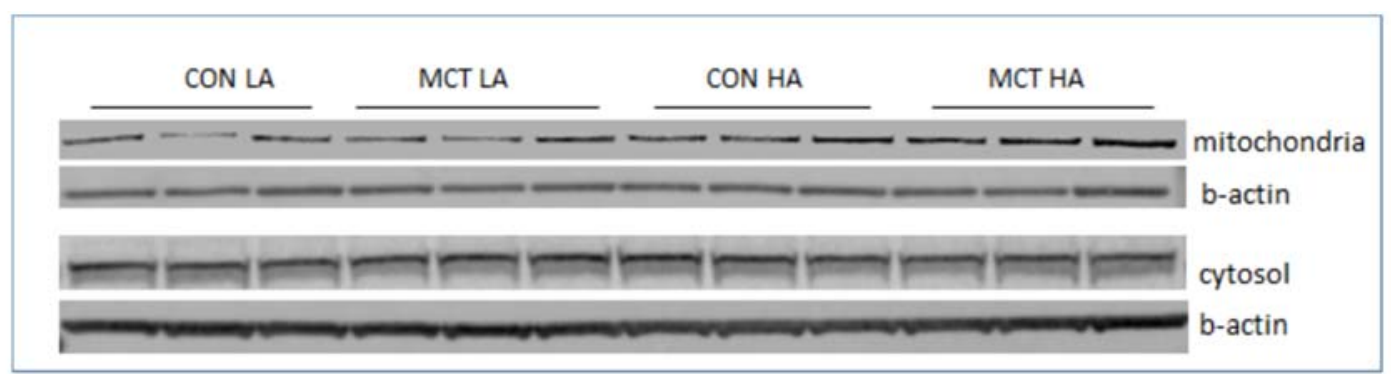

Supplementary Figure 4. Representative blots are included for each of the metabolic signaling pathway proteins investigated in the medial prefrontal cortex $(A)$ and for the mitochondrial vs cytosolic hexokinase protein levels (B). 